TRANSACTIONS OF THE

AMERICAN MATHEMATICAL SOCIETY

Volume 353, Number 5, Pages 1705-1739

S 0002-9947(00)02670-2

Article electronically published on December 18, 2000

\title{
VESSIOT STRUCTURE FOR MANIFOLDS OF $(p, q)$-HYPERBOLIC TYPE: DARBOUX INTEGRABILITY AND SYMMETRY
}

\author{
PETER J. VASSILIOU
}

\begin{abstract}
It is well known that if a scalar second order hyperbolic partial differential equation in two independent variables is Darboux integrable, then its local Cauchy problem may be solved by ordinary differential equations. In addition, such an equation has infinitely many non-trivial conservation laws. Moreover, Darboux integrable equations have properties in common with infinite dimensional completely integrable systems.

In this paper we employ a geometric object intrinsically associated with any hyperbolic partial differential equation, its hyperbolic structure, to study the Darboux integrability of the class $E$ of semilinear second order hyperbolic partial differential equations in one dependent and two independent variables. It is shown that the problem of classifying the Darboux integrable equations in $E$ contains, as a subproblem, that of classifying the manifolds of $(p, q)$ hyperbolic type of rank 4 and dimension $2 k+3, k \geq 2 ; p=2, q \geq 2$.

In turn, it is shown that the problem of classifying these manifolds in the two (lowest) cases $(p, q)=(2,2),(2,3)$ contains, as a subproblem, the classification problem for Lie groups. This generalizes classical results of E. Vessiot.

The main result is that if an equation in $E$ is $(2,2)$ - or $(2,3)$-Darboux integrable on the $k$-jets, $k \geq 2$, then its intrinsic hyperbolic structure admits a Lie group of symmetries of dimension $2 k-1$ or $2 k-2$, respectively. It follows that part of the moduli space for the Darboux integrable equations in $E$ is determined by isomorphism classes of Lie groups.

The Lie group in question is the group of automorphisms of the characteristic systems of the given equation which leaves invariant the foliation induced by the characteristic (or, Riemann) invariants of the equation, the tangential characteristic symmetries. The isomorphism class of the tangential characteristic symmetries is a contact invariant of the corresponding Darboux integrable partial differential equation.
\end{abstract}

\section{Introduction}

If a scalar second order hyperbolic partial differential equation in two independent variables is Darboux integrable, then its Cauchy problem can be solved by ordinary differential equations. Its "general solution" can be expressed in finite terms of arbitrary functions and their derivatives; it has infinitely many nontrivial conservation laws. As shown recently by Anderson and Kamran [AK], it has infinitely many higher-degree form-valued conservation laws as well. In addition, a Darboux integrable equation has linearizing Bianchi transformations. Moreover, a

Received by the editors November 17, 1998 and, in revised form, December 16, 1999.

2000 Mathematics Subject Classification. Primary 58D27, 58J45, 58J70; Secondary 35L70.

(C)2000 American Mathematical Society 
Darboux integrable equation has properties in common with completely integrable systems and is sometimes referred to as geometrically integrable.

The problem of characterizing the class of completely integrable systems is one of great importance within the theory of partial differential equations and has recently produced a great deal of significant research (see $[\mathrm{Ne}, \mathrm{Pa}$ ). The basic tenent of this paper is that within the class of completely integrable equations, the Darboux integrable ones are, in some sense, the simplest and "most accessible". Nevertheless, the task of classifying these equations in the present state of development appears to be very difficult. The first major contribution to the problem of classification was due to Goursat [Go99] who classified the hyperbolic semilinear partial differential equations in one dependent and two independent variables that are Darboux integrable on the 2-jets. Thus, in appropriate coordinates, the class of equations that Goursat considered are those of the form

$$
u_{x y}=f\left(x, y, u, u_{x}, u_{y}\right) .
$$

This class of equations, denoted by the symbol $E$, is the object of study in this paper. A slightly weaker version of Darboux integrability, so called Darboux semiintegrability (on the 2-jets), was studied by Cartan in his famous "five variables" paper [Ca] using his method of equivalence. In that paper, Cartan presented the first example of a Cartan geometry (see R. Sharpe, Sh] ). More precisely, to each second order scalar partial differential equation in two independent variables Darboux semi-integrable on the 2-jets one may associate a certain Pfaffian system on a five dimensional manifold, $M_{5}$, and a connection whose curvature is a certain rank 4 tensor, $\mathcal{T}$. The scale of the classification problem for Darboux semi-integrability on the 2-jets may be gauged by realising that the tensor $\mathcal{T}$ appears to play a role in this Cartan geometry that the Ricci tensor plays in Riemannian geometry $[\mathrm{BH}]$.

In this paper it is explained that every scalar second order hyperbolic partial differential equation in two independent variables is intrinsically associated with a geometric object referred to as a manifold of $(p, q)$-hyperbolic type (of rank 4) 1 Roughly speaking, this is a smooth manifold $M$, of dimension greater than or equal to 6 , together with a hyperbolic structure, $\mathcal{H}$ which is a rank 4 distribution on $M$ satisfying certain structure equations. The classification of these manifolds without the imposition of additional structure does not seem to be possible or, for that matter, desirable. For instance, in one of the simplest cases, when the dimension of $M$ is 7 , it contains the problem of classifying all scalar second order hyperbolic partial differential equation in two independent variables with respect to contact transformations. The resulting list of equations, if it could be obtained, would be so complicated that it would make little sense 2 However, following Vessiot, [Vess39, Vess42], we show that by imposing additional structure, a description of the resulting manifolds of hyperbolic type is indeed possible and that this description lay at the heart of the classification problem of Darboux integrability for the class E.

\footnotetext{
${ }^{1}$ The term 'manifold of hyperbolic type' used here is not related to the term 'hyperbolic manifold' used in other contexts.

${ }^{2}$ The beginnings of a study of the equivalence problem for scalar hyperbolic partial differential equation in two independent variables has been undertaken, for instance, in [GK] and in $[\mathrm{K}]$. There are numerous interesting results arising from branches of the problem exhibiting a high degree of symmetry. However, as the authors point out, the complexity of constructing a complete list of equivalence classes may be appreciated by consulting [HsK] where the corresponding problem is considered in the much simpler case of second order ordinary differential equations.
} 
The basic tools that we use are Vessiot's geometric formulation of differential equations, Vess24, and in particular his analysis of the Darboux integrability on the 2-jets of the equations $E$ Vess39. Vess42. Indeed, we revisit Vessiot's main structure theorem for manifolds of (2,2)- and (2,3)-hyperbolic type of rank 4 and dimension 7 and show that a similar result holds in any dimension greater than or equal to 6 , thereby obtaining local normal forms for these manifolds. Since each equation in $E$ that is either $(2,2)$ - or $(2,3)$-Darboux integrable on the $k$-jets 3 determines such a manifold, this normal form result has deep implications for the classification problem of the Darboux integrable equations in $E$ and in particular shows that Vessiot's analysis of partial differential equations in the class $E$ that are Darboux integrable on the 2-jets can be extended to the $k$-jets for all $k \geq 2$.

The key to the classification of, and normal form for, manifolds of $(2,2)$ - and $(2,3)$-hyperbolic type rests on the fact that the corresponding hyperbolic structures determine and are determined by Lie groups of each dimension greater than or equal to zero 4 It follows that isomorphism classes of Lie groups determine a part of the moduli space of these manifolds. This in turn implies that the classification problem for the set $\operatorname{Darb}(E) \subset E$, of Darboux integrable equations in $E$, contains as a subproblem, the classification problem for Lie groups. This extends the classical result of Vessiot who demonstrated that the classification problem on the 2-jets for $\operatorname{Darb}(E)$ depends upon the classification of the complex Lie groups of dimension 2 and 3, corresponding to the lowest case, $k=2$. Indeed, for $p=2$ and $q=2$ or 3 , the normal forms which constitute the generalization of these classical results prove that the differential equations manifold of a $(2,3)$ - or $(2,3)$-Darboux integrable equation in $E$ is locally equivalent to either $\mathbb{R}^{4} \times G_{2 k-1}$ or to $\mathbb{R}^{5} \times G_{2 k-2}$, respectively, for integers $k \geq 2$, where $G_{2 k-1}$ and $G_{2 k-2}$ are Lie groups of dimension $2 k-1$ and $2 k-2$ respectively.

It is shown that the Lie groups in question are realised as the groups of automorphisms of the characteristic systems. More precisely, if an equation in $E$ is either $(2,2)$ - or (2,3)-Darboux integrable at some order, then the symmetries of its characteristic systems which are tangent to the joint level sets of the characteristic invariants are precisely the Lie groups featured in Vessiot's structure theorem. It is shown that this result generalizes to all prolongations. We call these the tangential characteristic symmetries. Thus, the tangential characteristic symmetries enable us to 'see' the Lie groups featured in Vessiot's structure theorems. These symmetries play a role in the equivalence problem for $\operatorname{Darb}(E)$ since their isomorphism class is a contact invariant of the corresponding partial differential equation.

We remark that the main structure theorem of this paper is applicable to much more general classes of partial differential equations than equation class $E$. The restriction here to the semilinear case is not essential. For instance, in Va99] it has been applied to systems of two first order partial differential equations in two dependent and two independent variables. Finally, we note that a description of the rank 4, manifolds of $(p, q)$-hyperbolic type for $p=2, q \geq 4$ would complete the description of the full class $\operatorname{Darb}(E)$ of Darboux integrable equations in $E$.

\footnotetext{
${ }^{3}$ For instance, ' $(2,5)$-Darboux integrable on the 9-jets', means that at order 9 one characteristic system has 2 invariants and the other has 5. A detailed explanation is given in section 3 of this paper.

${ }^{4}$ There is a unique equivalence class of manifolds where the group in question consists only of the identity. Every such manifold is locally equivalent to the product of a pair of contact 3 -manifolds Va99]. See 6 for the definition of local equivalence.
} 
Throughout, all functions, vector fields, differential forms, etc., will be assumed to be smooth unless otherwise specified. All Pfaffian systems, vector field distributions, etc., will be assumed to have constant rank on their domains of definition.

\section{Vessiot Distributions of EQUATIONS in $E$ AND MANiFolds OF $(p, q)$-HYPERBOLIC TYPE OF RANK 4}

The class of equations, denoted by $E$, that comprise this study have the form

$$
u_{x y}=f\left(x, y, u, u_{x}, u_{y}\right),
$$

where $f$ is a smooth function of all its variables. Equation (2.1) defines a submanifold of the second order jet bundle $J^{2}\left(\mathbb{R}^{2}, \mathbb{R}\right)$, and denoted by $\mathcal{R}_{2}$. Of importance are the prolongations of equation (2.1). The first prolongation is obtained by differentiating (2.1) with respect to $x$ and $y$ to obtain an overdetermined system for the unknown function $u(x, y)$ :

$$
u_{x y}=f, \quad u_{x y x}=\frac{d f}{d x}, \quad u_{x y y}=\frac{d f}{d y} .
$$

Equation (2.2) defines a submanifold of the third order bundle $J^{2}\left(\mathbb{R}^{2}, \mathbb{R}\right)$ and denoted by $\mathcal{R}_{3}$. The $r$ th prolongation of equation (2.1) is obtained by appending to the $(r-1)$ st prolongation, all its derivatives with respect to $x$ and $y$. This defines a submanifold of the jet bundle of order $r+2$, for all $r \geq 0$ and denoted by $\mathcal{R}_{r+2}$.

Let $i_{k}: \mathcal{R}_{k} \rightarrow J^{k}\left(\mathbb{R}^{2}, \mathbb{R}\right)$ be the standard inclusion and $\Omega^{k}\left(\mathbb{R}^{2}, \mathbb{R}\right)$ the contact system on $J^{k}\left(\mathbb{R}^{2}, \mathbb{R}\right)$. From now on $J^{k}\left(\mathbb{R}^{2}, \mathbb{R}\right)$ will be denoted simply as $J^{k}$ and the contact system on $J^{k}$ as $\Omega^{k}$. Coordinates on $J^{k}$ will be denoted by $x, y, u, u_{p, q}, p, q=$ $0,1,2, \ldots, k ; p+q \leq k$, where

$$
u\left(j^{k} s\right)=s(x, y), \quad u_{p, q}\left(j^{k} s\right)=\frac{\partial^{p+q} s}{\partial x^{p} \partial y^{q}},
$$

and where $j^{k} s$ denotes the $k$-jet of a smooth real-valued function, $s$, on $\mathbb{R}^{2}$. The $k$ th order total derivative operators associated to equation (2.1), $D_{1}^{(k)}$ and $D_{2}^{(k)}$ are defined recursively by

$$
\begin{aligned}
& D_{1}^{(j+1)}=D_{1}^{(j)}+u_{j+1,0} \partial_{u_{j, 0}}+\left(D_{2}^{(j)}\right)^{j-1} f \partial u_{0, j}, \\
& D_{2}^{(j+1)}=D_{2}^{(j)}+\left(D_{1}^{(j)}\right)^{j-1} f \partial_{u_{j, 0}}+u_{0, j+1} \partial_{u_{0, j}},
\end{aligned}
$$

for $j=2,3, \ldots, k-1$, and

$$
\begin{gathered}
D_{1}^{(2)}=\partial_{x}+u_{1,0} \partial_{u}+u_{2,0} \partial_{u_{1,0}}+f \partial_{u_{0,1}}, \\
D_{2}^{(2)}=\partial_{y}+u_{1,0} \partial_{u}+f \partial_{u_{1,0}}+u_{0,2} \partial_{u_{0,1}} .
\end{gathered}
$$

Note that the total differential operators have the obvious meaning: if $F$ is a function on $\mathcal{R}_{k}$, and $s$ is a solution of (2.1), then the $D_{i}^{(k)}, i=1,2$, satisfy,

$$
\left(D_{i}^{(k+1)} F\right)\left(j^{k+1} s\right)=\frac{\partial}{\partial x^{i}} F\left(j^{k} s\right), \quad i=1,2 .
$$


Proposition 2.1. The distribution $F^{(k)}$ of vector fields $\left(i^{*} \Omega^{k}\right)^{\perp}$ is

$$
\left\{D_{1}^{(k)}, D_{2}^{(k)}, \partial_{u_{k, 0}}, \partial_{u_{0, k}}\right\}
$$

where braces denote the distribution spanned by the enclosed vector fields.

The significance of the distribution $F^{(k)}$ is given by Proposition 2.2 which, roughly speaking, states that real analytic local solutions of $\mathcal{R}_{k}$ are in one-to-one correspondence with Frobenius subdistributions $G_{2} \subset F^{(k)}$ such that projection to the base is the distribution $\left\{\partial_{x}, \partial_{y}\right\}$. More precisely, a local solution of $\mathcal{R}_{k}$ is a map $s: \mathbb{R}^{2} \mapsto \mathbb{R}$, whose $k$-graph, $j^{k} s$, defines a 2-dimensional submanifold of $\mathcal{R}_{k}$. If $\mathcal{D}$ is a differential system on a manifold $M$, a submanifold $N \subset M$ is an integral submanifold of $\mathcal{D}$ if and only if $T_{p} N \subset \mathcal{D}_{p}^{\perp}$, for all $p \in N$, where $\mathcal{D}_{p}^{\perp}$ is the value of the distribution $\mathcal{D}^{\perp}$ at $p$. Equivalently, if $\phi: N \rightarrow M$ is a one to one immersion, then $\phi^{*} \mathcal{D}=0$. A submanifold $S \subset \mathcal{R}_{k}$ is a solution submanifold of the $(k-2)$-times prolongation of equation (2.1), if and only if $i_{k}(S) \subset J^{k}$ is the image of the $k$-graph of a function $s: \mathbb{R}^{2} \mapsto \mathbb{R}$. The following proposition characterizes all analytic solution submanifolds of $\mathcal{R}_{k}$ when $f$ is an analytic function. That is, those which arise as the $k$-graphs of analytic functions $s$ or are cut out by analytic functions on $\mathcal{R}_{k}$.

Proposition 2.2. An analytic submanifold $S \subset \mathcal{R}_{k}$, where $\mathcal{R}_{k}$ is analytic, is a solution submanifold if and only if there is a Frobenius integrable subdistribution $G_{2} \subset F^{(k)}$ such that $S$ is a leaf of the foliation of $G_{2}$ and $\alpha_{*}\left(G_{2}\right)=\left\{\partial_{x}, \partial_{y}\right\}$, where $\alpha$, denotes the source projection $J^{k} \mapsto \mathbb{R}^{2}$.

Proof. Suppose that $\mathcal{R}_{k}$ is analytic (that is, $f$ in (2.1) is an analytic function on $J^{1}$ ) and $S \subset \mathcal{R}_{k}$ is an analytic solution submanifold. It is easy to see that $T_{p} S \subset$ $\left(i_{k}^{*} \Omega^{k}\right)_{p}^{\perp}=F_{p}^{(k)}$. It follows from Vessiot's proof of the Cartan-Kähler theorem Vess24, that there is a Frobenius integrable subdistribution $G_{2} \subset F^{(k)}$ such that $T_{p} S=\left(G_{2}\right)_{p}$ and $\alpha_{*} G_{2}=\left\{\partial_{x}, \partial_{y}\right\}$. This implies that, at least in the analytic case, the solution $S$ may be "embedded" in a local foliation of $\mathcal{R}_{k}$. Conversely, if $G_{2} \subset F^{(k)}$ is a 2-dimensional Frobenius subdistribution which projects and $S$ is a leaf of the foliation, then from the definitions it follows that $S$ is a solution submanifold.

Thus, the problem of finding all the local analytic solutions of the $(k-2)$-times prolongation of equation (2.2) is equivalent to finding all of the analytic solution submanifolds of $\mathcal{R}_{k}$, which, in turn, is equivalent to finding all the "projectible", Frobenius integrable subdistributions of $F^{(k)} 5$ The distribution $F^{(k)}$ will be called the Vessiot distribution of, or, associated to, the differential equation $\mathcal{R}_{k}$. Vessiot Vess24, has given an algorithm for constructing all the Frobenius integrable subdistributions of any given distribution; the structure equations play a fundamental role. In this work, the structure equations of $F^{(k)}$ are systematically exploited to obtain information on the Darboux integrable equations within the class of equations $E 6$

\footnotetext{
${ }^{5}$ Note that in the exterior differential systems formulation of the Cartan-Kähler theorem, the distribution $F^{(k)}$ defines the collection of 1-integral elements.

${ }^{6}$ Because of the limitations of the Cauchy-Kovalevsky theorem upon which the Cartan-Kähler theorem depends, the requirement of analyticity in Proposition 2.2 is generally unavoidable. There are instances, however, where analyticity can be weakened to smoothness; for instance, for the class of equations treated in this paper. See 3
} 
Proposition 2.3. The structure equations in $F^{(k)}$ are

$$
\begin{gathered}
{\left[D_{1}^{(k)}, D_{2}^{(k)}\right]=\left(\left(D_{1}^{(k)}\right)^{k-1} f\right) \partial_{u_{k-1,0}}-\left(\left(D_{2}^{(k)}\right)^{k-1} f\right) \partial_{u_{0, k-1}},} \\
{\left[D_{1}^{(k)}, \partial_{u_{k, 0}}\right]=-\partial_{u_{k-1,0}},\left[D_{1}^{(k)}, \partial_{u_{0, k}}\right]=0,} \\
{\left[D_{2}^{(k)}, \partial_{u_{k, 0}}\right]=0,\left[D_{2}^{(k)}, \partial_{u_{0, k}}\right]=-\partial_{u_{0, k-1}} .}
\end{gathered}
$$

Proof. By induction on $k$, making use of the recurrence relations (2.3) for the total differential operators. Case $k=2$ is direct easy calculation. A calculation and making use of the inductive hypothesis show that

$$
\left[D_{1}^{(j+1)}, D_{2}^{(j+1)}\right]=D_{1}^{(j+1)}\left(D_{1}^{(j)}\right)^{j-1} f \partial_{u_{j, 0}}-D_{2}^{(j+1)}\left(D_{2}^{(j)}\right)^{j-1} f \partial_{u_{0, j}} .
$$

Using the fact that $f$ is a first order differential function, and another induction, shows that $D_{i}^{(j+1)}\left(D_{i}^{(j)}\right)^{j-1} f=\left(D_{i}^{(j+1)}\right)^{j} f, i=1,2$, establishing the result for the Lie bracket of the total differential operators. The remaining structure equations are easily checked.

Classically, a fundamental feature of hyperbolic partial differential equations is the existence of characteristics as explained in standard texts such as Courant and Hilbert $[\mathrm{CH}]$. On the other hand, any Pfaff system or equivalently, vector field distribution has, associated with it, an important geometric object, its characteristic variety. Not surprisingly, the classical notion of characteristics for partial differential equations and that of characteristic variety of associated differential systems are very closely related and play an extremely important role; see, for instance, BC3G], $\mathrm{BGH}$. In this paper we will study the geometry of the equation class $E$ by studying the Vessiot distributions $F^{(k)}$ canonically associated to prolongations of equations in $E$. We will therefore confine our discussion of the characteristic variety to that of vector field distributions. The reader is directed to the above cited references for alternative and more general discussions.

Let $\mathcal{F}$ be a distribution of vector fields. The degree of $X \in \mathcal{F}$ is the integer

$$
\operatorname{dim}([X, \mathcal{F}] / \mathcal{F}) .
$$

Clearly, if $\mathcal{F}$ is Frobenius, then every vector field in $\mathcal{F}$ has degree zero. An elementary but important fact is that the set of degree zero vector fields in $\mathcal{F}$ is a Frobenius integrable subdistribution called the Cauchy system, denoted $\operatorname{char}(\mathcal{F})$. The structure equations recorded in Proposition 2.3 show that the degree of the generic vector fields in $F^{(k)}$ is two. But there may be nongeneric, or singular vector fields with degree less than two. These, if they exist, turn out to be of central importance for the study of the solutions of equation (2.1). The following describes all the singular vector fields in $F^{(k)}$.

Proposition 2.4. The Vessiot distribution $F^{(k)}$ has no (nontrivial) degree zero vector fields. A nonzero vector field $X \in F^{(k)}$ has degree one if and only if

$$
X \in\left\{D_{1}^{(k)}+\left(\left(D_{2}^{(k)}\right)^{k-1} f\right) \partial_{u_{0, k}}, \partial_{u_{k, 0}}\right\}=\left\{X_{1}^{(k)}, X_{3}^{(k)}\right\}=F_{1}^{(k)},
$$

or,

$$
X \in\left\{D_{2}^{(k)}+\left(\left(D_{1}^{(k)}\right)^{k-1} f\right) \partial_{u_{k, 0}}, \partial_{u_{0, k}}\right\}=\left\{X_{2}^{(k)}, X_{4}^{(k)}\right\}=F_{2}^{(k)} .
$$


Proof. Let $A=a_{1} D_{1}^{(k)}+a_{2} D_{2}^{(k)}+a_{3} \partial_{u_{k, 0}}+a_{4} \partial_{u_{0, k}}, B=b_{1} D_{1}^{(k)}+b_{2} D_{2}^{(k)}+b_{3} \partial_{u_{k, 0}}+$ $b_{4} \partial_{u_{0, k}}$, be arbitrary vector fields in $F^{(k)}$. Making use of the structure equations of Proposition 2.3, shows that

$$
[A, B] \equiv 0 \quad \bmod F^{(k)},
$$

if and only if

$$
\left(\begin{array}{cccc}
a_{3}-a_{2}\left(D_{1}^{(k)}\right)^{k-1} f & a_{1}\left(D_{1}^{(k)}\right)^{k-1} f & -a_{1} & 0 \\
a_{2}\left(D_{2}^{(k)}\right)^{k-1} f & a_{4}-a_{1}\left(D_{2}^{(k)}\right)^{k-1} f & 0 & -a_{2}
\end{array}\right)\left(\begin{array}{l}
b_{1} \\
b_{2} \\
b_{3} \\
b_{4}
\end{array}\right)=0
$$

where $A$ is regarded as a fixed vector field and $B$ is to be determined so as to satisfy (2.4). Denote by $M_{A}$ the $2 \times 4$ matrix featured in (2.5). If there is a vector field $A \neq 0$ so that $M_{A}$ has rank zero, then (2.5) has a 4-dimensional solution space and therefore $A$ stabilizes $F^{(k)}$. This implies that $A$ is a nontrivial characteristic (i.e. Cauchy) vector field for $F^{(k)}$. By inspection of equation (2.5) we see that $\operatorname{char}\left(F^{(k)}\right)=0$. That is, there are no (nontrivial) vector fields of degree zero. We now seek degree 1 vector fields in $F^{(k)}$. If there is a vector field $A$ such that $M_{A}$ has rank 1 , then the system (2.5) has a 3 -dimensional solution space and consequently there is a 1-dimensional subdistribution not stabilized by $A$. Hence $A$ has degree 1. Thus, to determine the degree 1 vector fields it is enough to determine those $A$ such that $M_{A}$ has rank 1. Suppose $a_{1} \neq 0$. Then the third column of $M_{A}$ is nonzero and hence all the other columns are multiples of it; thus, $a_{2}=0$. Also, the vanishing of the determinant of the $2 \times 2$ matrix formed from columns 2 and 3 gives $a_{4}=a_{1}\left(D_{2}^{(k)}\right)^{k-1} f$. It is now easily checked that with this choice of $a_{4}$, the determinant of the matrix formed from columns 1 and 2 is also zero. Thus, if $a_{1} \neq 0$, the matrix will have rank 1 if and only if

$$
A=a_{1} D_{1}^{(k)}+a_{3} \partial_{u_{k-1,0}}+a_{1}\left(D_{2}^{(k)}\right)^{k-1} f \partial_{u_{0, k-1}} .
$$

In short, since $a_{3}$ is arbitrary, we have that if $a_{1} \neq 0$, then $A$ has degree 1 if and only if $A \in F_{1}^{(k)}$.

Suppose $a_{1}=0$. Then the matrix in (2.5) has the form

$$
\left(\begin{array}{cccc}
a_{3}-a_{2}\left(D_{1}^{(k)}\right)^{k-1} f & 0 & 0 & 0 \\
a_{2}\left(D_{2}^{(k)}\right)^{k-1} f & a_{4} & 0 & -a_{2}
\end{array}\right) .
$$

Here there are two cases to consider according as $a_{2} \neq 0, a_{2}=0$. If $a_{2} \neq 0$, then the last column of $M_{A}$ is nonzero and the matrix $M_{A}$ will have rank 1 if and only if the $2 \times 2$ matrix formed from columns 1 and 4 of (2.6) has determinant zero giving the relation $a_{3}=a_{2}\left(D_{2}^{(k)}\right)^{k-1} f$. Thus, in this case a vector field $A \in F^{(k)}$ has degree 1 if and only if $A \in F_{2}^{(k)}$. If $a_{2}=0$, then (2.6) will have rank 1 if and only if exactly one of $a_{3}, a_{4}$ is zero. If $a_{3}=0, a_{4} \neq 0$, then $A \in\left\{\partial_{u_{k, 0}}\right\}$. If $a_{4}=0$, $a_{3} \neq 0$, then $A \in\left\{\partial_{u_{0, k}}\right\}$.

We may now say how the notion of a singular vector field belonging to a given distribution $\mathcal{F}$ is related to the characteristic variety of $\mathcal{F}$. Now for any nonzero function $\lambda$ on $M$ and any $X \in \mathcal{F}$, the degree of $X$ and $\lambda X$ agree; from which it follows that the notion of degree is really "projective". More precisely, let us denote by $\pi: G_{1}(\mathcal{F}) \rightarrow M$, the Grassmann bundle of 1-planes over $M$ that lie in $\mathcal{F}$. A section of $\pi$ is a line field and any element $X \in \mathcal{F}$ gives rise to a line field $[X]$ on 
$M$. We are naturally led to define a map $\operatorname{deg}_{\mathcal{F}}: \Gamma(\pi) \rightarrow \mathbb{N}$ on the space of sections $\Gamma(\pi)$ of $\pi$ by

$$
\operatorname{deg}_{\mathcal{F}}([X])=\operatorname{dim}([X, \mathcal{F}] / \mathcal{F})
$$

which is well defined. In the language of exterior differential systems, the point $\left[X_{p}\right]$ lying in the fibre $\pi^{-1}(p)$ is called a 1-integral element and often denoted by the symbol $E_{p}^{1}$. For the distribution of interest $F^{(k)}$, we see from the structure equations that $\operatorname{deg}_{F^{(k)}}$ generically has value 2. Proposition (2.4) shows that $\operatorname{deg}_{F^{(k)}}$ takes value zero if and only if $[X]=0$. It takes value 1 if and only if $[X]$ lies in either $\mathbb{P} F_{1}^{(k)}$ or $\mathbb{P} F_{2}^{(k)}$, where $\mathbb{P} V$ denotes the projectivisation of linear space $V$. Thus, $\operatorname{deg}_{F^{(k)}}$ induces a fibration over the differential equations manifold $\left(\mathcal{R}_{k}, F^{(k)}\right)$ whose typical fibre may be identified with the union of two projective lines: $\mathbb{P} \cup \mathbb{P}$. All this may be informally expressed by saying that the function $\operatorname{deg}_{F^{(k)}}$ takes its minimum value on a projective subvariety of the space of 1-integral elements of $F^{(k)}$ and that this subvariety may be identified with $\mathbb{P} \cup \mathbb{P}$. This what we refer to as the characteristic variety of $F^{(k)}, \mathcal{C}\left(F^{(k)}\right)$. We have

$$
\mathcal{C}\left(F^{(k)}\right)=\mathbb{P} F_{1}^{(k)} \cup \mathbb{P} F_{2}^{(k)} .
$$

It is easy to see that $F^{(k)}$ may be expressed in a basis of singular (degree one) vector fields. The structure of $F^{(k)}$ in this basis is most important.

Proposition 2.5. The structure equations in $F^{(k)}=F_{1}^{(k)}+F_{2}^{(k)}$ are

$$
\left[F_{1}^{(k)}, F_{1}^{(k)}\right] \equiv \partial_{u_{k-1,0}}, \quad\left[F_{1}^{(k)}, F_{2}^{(k)}\right] \equiv 0, \quad\left[F_{2}^{(k)}, F_{2}^{(k)}\right] \equiv \partial_{u_{0, k-1}} \quad \bmod F^{(k)}
$$

In view of the structure equations of Proposition [2.5, we regard $F^{(k)}$ as decomposable into a direct sum of singular distributions,

$$
F^{(k)}=F_{1}^{(k)} \oplus F_{2}^{(k)} .
$$

We note that this direct sum structure is equivalent to hyperbolicity in the corresponding partial differential equation. We will prove this in the more general context of arbitrary second order partial differential equations in one dependent and two independent variables of locally constant type. Recall that

$$
H\left(x, y, u, u_{x}, u_{y}, u_{x x}, u_{x y}, y_{y y}\right)=0,
$$

is said to be elliptic, parabolic, or hyperbolic at a point $p$ on the submanifold, $\mathcal{R}$, defined by $H=0$ if and only if at $p$, the quantity $\Delta=\left(H_{u_{12}}\right)^{2}-4 H_{u_{11}} H_{u_{22}}$ is negative, zero or positive, respectively.

Proposition 2.6. Let $\mathcal{F}$ be the Vessiot distribution of a second order partial differential equation $H=0$ in one dependent and two independent variables of locally constant type. Then $H=0$ is hyperbolic at $p \in \mathcal{R}$ if and only if there is a neighborhood of $p$ on which $\mathcal{F}$ decomposes as a direct sum of rank 2 subdistributions $\mathcal{F}_{1}$ and $\mathcal{F}_{2}$ satisfying the structure equations

$$
\left[\mathcal{F}_{1}, \mathcal{F}_{1}\right] \equiv Z_{1},\left[\mathcal{F}_{1}, \mathcal{F}_{2}\right] \equiv 0,\left[\mathcal{F}_{2}, \mathcal{F}_{2}\right] \equiv Z_{2} \quad \bmod \mathcal{F}
$$

for some linearly independent vector fields $Z_{1}, Z_{2}$.

Proof. It is always possible to express a second order partial differential equation $H=0$ in the form

$$
u_{y y}=F\left(x, y, u, u_{x}, u_{y}, u_{x x}, u_{x y}\right)
$$


for some $F$, possibly after a change of independent variables. This is a change of coordinates on the submanifold $\mathcal{R}$ defined by $H=0$. The Vessiot distribution $\mathcal{F}$ for (2.8) is spanned by the vector fields

$$
\begin{array}{cl}
X_{1}=\partial_{x_{1}}+u_{1} \partial_{u}+u_{11} \partial_{u_{1}}+u_{12} \partial_{u_{2}}, & X_{2}=\partial_{x_{2}}+u_{2} \partial_{u}+u_{12} \partial_{u_{1}}+F \partial_{u_{2}}, \\
X_{3}=\partial_{u_{12}}, & X_{4}=\partial_{u_{11}} .
\end{array}
$$

Suppose $H=0$ is hyperbolic. By calculations similar to those of Proposition 2.4 we deduce that a nonzero vector field $X \in \mathcal{F}$ has degree 1 if and only if

$$
X \in\left\{X_{2}+\lambda_{1} X_{1}+\left(X_{1} F\right) X_{3}, X_{4}+\lambda_{2} X_{3}\right\}=\mathcal{F}_{1},
$$

or

$$
X \in\left\{X_{2}+\lambda_{2} X_{1}+\left(X_{1} F\right) X_{3}, X_{4}+\lambda_{1} X_{3}\right\}=\mathcal{F}_{2},
$$

where $\lambda_{1}, \lambda_{2}$ are the two real roots of

$$
\lambda^{2}+F_{u_{12}} \lambda-F_{u_{11}}=0 .
$$

From our previous discussion this shows that the characteristic variety $\mathcal{C}(\mathcal{F})=$ $\mathbb{P} \mathcal{F}_{1} \cup \mathbb{P} \mathcal{F}_{2}$. One checks by direct calculation that $\mathcal{F}_{1}, \mathcal{F}_{2}$ satisfy the claimed structure equations.

Conversely, suppose one is in a region $U$ and $\mathcal{R}$ in which the equation is not hyperbolic. Hence on $U, \Delta \leq 0$. If $\Delta<0$, then the argment is formally identical to the one in the hyperbolic case except that equation (2.9) has no real roots, so there are no degree 1 vector fields at all. If $\Delta=0$ on $U$, then arguing as above shows that $X$ has degree 1 if and only if it is a nonzero element of $\left\{X_{2}+\lambda X_{1}+\right.$ $\left.\left(X_{1} F\right) X_{3}, X_{4}-\lambda X_{3}\right\}$, where $\lambda$ is the single real root of (2.9). This time there is only a rank 2 subdistribution of degree 1 vector fields. As a hyperbolic basis is made up of degree 1 vector fields the result follows.

Proposition 2.6 shows that the hyperbolicity of a second order scalar partial differential equation in two independent variables is invariant under contact transformations. Gardner [Ga] has given a proof of this fact based on his symmetric conformal bilinear form. Using this, Gardner and Kamran [GK] made an extensive study of exterior differential systems satisfying hyperbolic structure equations. Among their results they essentially give the following.

Proposition 2.7 (Gardner and Kamran Ga], GK]). If a scalar second order partial differential equation in two independent variables, $H=0$, is hyperbolic at a point $p \in \mathcal{R}$, then there are generators $\omega^{1}, \pi^{1}, \pi^{2}$ of $I_{H}=\mathcal{F}^{\perp}$ on a neighbourhood $\mathcal{N}(p)$ of $p$ which extends to a coframe $I_{H}+\left\{\omega^{4}, \omega^{5}, \omega^{5} \omega^{7}\right\}$ on $\mathcal{N}(p)$ satisfying the structure equations

$$
\begin{aligned}
& d \omega^{1} \equiv 0 \\
& d \pi^{1} \equiv \omega^{4} \wedge \omega^{5}, \quad \bmod \left\{\omega^{1}, \pi^{1} \pi^{2}\right\} \\
& d \pi^{2} \equiv \omega^{6} \wedge \omega^{7} .
\end{aligned}
$$

Note that structure equations (2.10) and those of Proposition (2.6) are equivalent. For, if a distribution $\mathcal{F}$ on a 7 -dimensional manifold $M_{7}$ has hyperbolic basis $\mathcal{F}_{1}=\left\{X_{1}, X_{3}\right\}, \mathcal{F}_{2}=\left\{X_{2}, X_{4}\right\}$ we may define a coframe $\pi^{1}, \pi^{2}, \pi^{3}, \omega^{1}, \omega^{2}, \omega^{3}, \omega^{4}$ on $M_{7}$ by $\left\{\pi^{1}, \pi^{2}, \pi^{3}\right\}=\mathcal{F}^{\perp},\left\{\omega^{1}, \omega^{2}, \omega^{3}, \omega^{4}\right\}=\left\{Z_{1}, Z_{2}, Z_{3}\right\}^{\perp}, \pi^{i}\left(Z_{j}\right)=-\delta_{i j}$, $\omega^{\alpha}\left(X_{\beta}\right)=\delta_{\alpha \beta}$, where $Z_{3}$ is any vector field completing $\mathcal{F}+\left\{Z_{1}, Z_{2}\right\}$ to a frame on $M_{7}$. An easy computation using Cartan's formula $d \omega(X, Y)=X(\omega(Y))-$ 
$Y(\omega(X))-\omega([X, Y])$ verifies the claim. Thus, Proposition [2.6 verifies that the converse of Proposition [2.7 holds. Moreover, the systems studied in this paper (and first studied in [Vess39]) may be regarded as prolongations of certain of the Gardner-Kamran systems studied in [GK]. It is important to note that Proposition 2.6 shows that hyperbolicity is an intrinsic property of the differential equation. We say that a pair $(M, \mathcal{F})$ consisting of a smooth manifold $M$ and a distribution of vector fields $\mathcal{F}$ on $M$, is a differential equations manifold of a differential equation if there is a local diffeomorphism $\psi: M \rightarrow \Sigma$ such that $\psi_{*} \mathcal{F}=i^{*} \Omega^{\perp}$, where $\Omega$ is the contact system on some jet bundle $J$ and $i(\Sigma)$ is an immersed submanifold of $J$. That is, $\mathcal{F}^{\perp}$ is locally diffeomorphic to the restriction of $\Omega$ to an (immersed) submanifold of $J$, (see [KV]). Then we have

Theorem 2.8. Let $\mathcal{H}$ be a rank 4 distribution on a 7 dimensional manifold $M_{7}$ satisfying the structure equations of Proposition [2.6. Then $\left(M_{7}, \mathcal{H}\right)$ is the differential equations manifold of a scalar second order hyperbolic partial differential equation in two independent variables if and only if $\operatorname{dim}\left(\mathcal{H}^{\prime \prime}\right)=7$, $\operatorname{dim} \operatorname{char}\left(\mathcal{H}^{\prime}\right)=2$ and $\operatorname{char}\left(\mathcal{H}^{\prime}\right) \subset H$.

The proof follows directly from Proposition 2.6 together with the following theorem of Vessiot [Vess36].

Theorem 2.9 (E. Vessiot). Let $\mathcal{D}$ be a rank 4 distribution on a 7-dimensional manifold $M_{7}$. Then $\left(M_{7}, \mathcal{D}\right)$ is the differential equations manifold of a scalar second order partial differential equation in two independent variables if and only if

1. $\operatorname{dim}\left(\mathcal{D}^{\prime}\right)=6, \operatorname{dim}\left(\mathcal{D}^{\prime \prime}\right)=7$,

2. $\operatorname{dim} \operatorname{char}(\mathcal{D})=0, \operatorname{dim} \operatorname{char}\left(\mathcal{D}^{\prime}\right)=2, \operatorname{char}\left(\mathcal{D}^{\prime}\right) \subset \mathcal{D}$.

One consequence of this is that to study hyperbolic partial differential equations we may equivalently study manifolds upon which is defined a distribution satisfying the structure equations of Proposition 2.6, or equivalently, a Pfaff system satisfying (2.10). Finally, we note that Proposition 2.4 and the structure equations of Proposition 2.5 prove that the Vessiot distribution of every prolongation of equation (2.1) has a hyperbolic basis (consisting of degree 1 vector fields). Thus we are led to study distributions of vector fields on manifolds endowed with a hyperbolic structure.

Definition 2.10. A manifold of $(p, q)$-hyperbolic type of rank 4 is a pair $(M, \mathcal{H})$ consisting of

1. A smooth manifold $M$ of dimension at least 6 ,

2. A $(p, q)$-hyperbolic structure of rank $4, \mathcal{H}$, on $M$, meaning

(a) $\mathcal{H}$ is a rank 4 distribution on $M$,

(b) $\mathcal{H}$ has no regular invariants, that is, there is no regular function $\phi$ on $M$ such that $\mathcal{H}(\phi)=0$

(c) $\mathcal{H}$ decomposes as the direct sum of a pair of rank 2 subdistributions $\mathcal{H}_{1}$ and $\mathcal{H}_{2}$ satisfying the structure equations

$$
\left[\mathcal{H}_{1}, \mathcal{H}_{1}\right] \equiv Z_{1},\left[\mathcal{H}_{1}, \mathcal{H}_{2}\right] \equiv 0,\left[\mathcal{H}_{2}, \mathcal{H}_{2}\right] \equiv Z_{2}, \quad \bmod \mathcal{H}
$$

where $\left\{Z_{1}, Z_{2}\right\}$ is a rank 2 distribution on $M$.

(d) The subdistribution $\mathcal{H}_{1}$ has precisely $p$ functionally independent invariants and $\mathcal{H}_{2}$ has precisely $q$ functionally independent invariants. 
Remark. Condition (2b) implies that the final distribution in the derived flag of $\mathcal{H}$ has rank equal to the dimension of $M$. Manifolds of hyperbolic type of ranks other than 4 and dimension 7 occur in other applications. Rank 4 manifolds of hyperbolic type of dimension 6 occur in the study of systems of two first order partial differential equations in two independent variables Va99. The manifolds of hyperbolic type studied in this paper have rank 4 and dimension $2 k+3$, for $k \geq 2$. The case $k=2$ corresponds to second order scalar hyperbolic partial differential equations in the plane. The generic branch corresponds to the manifolds of $(0,0)$ hyperbolic type of rank 4 and dimension 7 . The sine-Gordon equation, $u_{x y}=\sin u$, is associated with a manifold of (1,1)-hyperbolic type of rank 4 and dimension 7 . The Liouville equation, $u_{x y}=e^{u}$, is associated with a manifold of (2,2)-hyperbolic type of rank 4 and dimension 7 . Lie's theorem, [Lie, GK], on the contact orbit of the wave equation may be expressed by saying that every manifold of $(3,3)$ hyperbolic type of rank 4 and dimension 7 is locally equivalent to the one associated to the linear wave equation $u_{x y}=0$. We will elaborate further on these remarks in the subsequent sections of this paper.

\section{Darboux integrability of the Equations $E$}

We may think of the hyperbolic structure associated with a hyperbolic partial differential equation as defining a partial moving frame on the differential equations manifold, $\mathcal{R}$, adapted to the geometry of the equation. In the next section this adapted frame will be studied to reveal some important symmetries associated with certain equations of $E$, those which are Darboux integrable and denoted by the symbol $\operatorname{Darb}(E)$ in $₫ 1$. In this section, we review some basic facts concerning the class of equations $\operatorname{Darb}(E)$ within the context of the previous section. This will establish some key results, provide context for the main results of this paper as well as providing a modern setting for this classical topic. We begin by linking the distributions $F_{1}^{(k)}, F_{2}^{(k)}$ of Proposition 2.4 to the standard notions of characteristics of hyperbolic equations.

If $s: \mathbb{R}^{2} \rightarrow \mathbb{R}$ is any smooth solution of equation (2.1), then an easy calculation shows that $\left(j^{k} s\right)_{*}\left(\partial_{x}\right) \in F_{1}^{(k)},\left(j^{k} s\right)_{*}\left(\partial_{y}\right) \in F_{2}^{(k)}$. This shows that each smooth solution admits two foliations by one-dimensional leaves arising as integral curves of $F_{1}^{(k)}$ and $F_{2}^{(k)}$, respectively. One may show that these foliations are transverse. Thus, we arrive at the familiar picture associated with hyperbolic partial differential equations in the plane, namely, a double foliation of each solution by curves, classically called Monge characteristics. Within the geometric framework that we have been describing, these arise as the integral curves of the singular subdistributions $F_{1}^{(k)}$ and $F_{2}^{(k)}$. For an alternative but related view of these issues the reader is referred to the works of Gardner and Kamran GK]; Bryant, Griffiths and Hsu BGH] and Anderson and Kamran [AK]. Indeed, in [GK], the authors have made an extensive study of the characteristic systems of order 2 associated with scalar second order hyperbolic partial differential equations in the plane based on the hyperbolic structure equations (2.10). In view of these facts, we will usually refer to the subdistributions $F_{1}^{(k)}$ and $F_{2}^{(k)}$ as the characteristic systems of order $k$; we expect the solvability properties of a given partial differential equation in $E$ to be strongly related to the properties of its characteristic systems. Indeed, the central object under study, Darboux integrability, may be most conveniently so expressed. 
Definition 3.1. A partial differential equation (2.1) is said to be Darboux integrable at order $k$ or Darboux integrable on the $k$-jets, $k \geq 2$, if each of its characteristic systems $F_{1}^{(k)}, F_{2}^{(k)}$ have at least two independent invariants and $k$ is minimal. They are called characteristic invariants of order $k$.

Before providing motivation for this definition, as well as giving some of its consequences, we will review a number of classical results that will be relevant to the task of this paper. In his extensive study of Darboux integrable equations [Go96, Goursat gave a characterization of the possible number of independent invariants that can occur on a characteristic system of any given order for an arbitrary second order partial differential equation in one dependent and two independent variables. We refer the reader to $[\mathrm{AK}]$ for a summary of Goursat's characterization and to the recent work of Juráš and Anderson [JA], where an invariant formulation of Goursat's results is given. Since we are only dealing with the class of equations $E$, the full details of Goursat's results will not be directly relevant. In our case it suffices to observe that $x$ and $y$ are invariants of $F_{2}^{(k)}$ and $F_{1}^{(k)}$, respectively. For an equation in $E$ to be Darboux integrable at order $k$, it suffices that a second invariant appear on each characteristic system after a certain number of prolongations. Proposition 3.2. proved below, shows that if one of the characteristic systems, say, $F_{1}^{(k)}$ acquires a second invariant at order $k$, then $F_{1}^{(k+i)}$ will have $2+i$ independent invariants, for all $i \geq 0$. This fact was first proved by Goursat Go96, in the context of arbitrary second order partial differential equations in one dependent and two independent variables and is proved below for the class of semilinear equations $E$ using the formulation of $\sqrt{2}$ of this paper. Moreover, it follows from Goursat's theorem (see [AK], $\S 6$; JA], $\S 5$ ), that Proposition 3.2 accounts for almost all of the invariants that can occur on the characteristic systems of the equations in $E$. That is, in general, at each prolongation, at most one further invariant may be acquired by a characteristic system. More precisely, the configurations of characteristic invariants that can occur for the equations in $\operatorname{Darb}(E)$ fall into a generic class and three exceptional classes. The exceptional classes are the (contact orbits of) the equations

$$
u_{x y}=u_{y} /(x+y), u_{x y}=u_{y} e^{u}
$$

and

$$
u_{x y}=0 .
$$

Using the same notation as in [AK], equations (3.1) fall into Goursat's class IIa. For equations (3.1a), one of the characteristic systems of order two has three independent invariants and the other two. Only one of these invariants has order two. The characteristic systems of order two for equation (3.1b) have three independent invariants each. Again, on either characteristic system, only one of the three invariants has order two. It is a theorem of Lie's Lie that equation (3.1b) defines the unique contact equivalence class of scalar second order partial differential equations in the plane with this property. This result is reproved, using geometric techniques in Vessiot [Vess39] and in Gardner and Kamran [GK]. That equations (3.1a) define the unique contact equivalence classes in $E$ with these properties is due to Goursat Go99. It had already been proved in [Go96] that the number of characteristic invariants on the 2-jets that can occur for any scalar second order hyperbolic partial differential equation in the plane is at most three. Proofs of all these facts based on the associated Vessiot distribution was given by Vessiot in [Vess39]. 
The remaining equations in $\operatorname{Darb}(E)$ fall into Goursat's class I where there is a characteristic system of minimal order, say, $k_{0}$ such that every characteristic invariant (of that order) is a function of either $x$ or $y$ and an invariant of order $k_{0} \geq 2$. It follows from these facts that for every equation in $\operatorname{Darb}(E)$, other than the above noted exceptions, there is a smallest order such that the 'configuration' of independent invariants on the characteristic systems is determined by an integer, $q \geq 2$, where $q$ is the number of invariants on one of the characteristic systems when the other one has acquired two independent invariants exactly. Thus, other than for the exceptional classes determined by equations (3.1), the "minimal configuration" of invariants for any Darboux integrable equation in $E$ is of the form $(2, q)$, meaning two invariants on one characteristic system and $q \geq 2$ on the other. If this $(2, q)$ configuration occurs at minimal order $k$, then we shall say that the equation is $(2, q)$-Darboux integrable on the $k$-jets. If we wish to emphasize that the equation is $(2, q)$-Darboux integrable at some order, then we will simply say that is it $(2, q)$ Darboux integrable. It follows from Proposition 3.2 that if an equation in $\operatorname{Darb}(E)$ has the $(2, q)$ configuration at order $k$, then it will have configuration $(3, q+1)$ at order $k+1$; etc.

Proposition 3.2. If $k \geq 2$ and $\phi_{j}$ is a kth order invariant of $F_{1}^{(k)}$, then $\phi_{k+1}=$ $D_{2}^{(k+1)} \phi_{k}$ is a $(k+1)$ st order invariant of $F_{1}^{(k+1)}$. Similarly, if $\psi_{k}$ is a kth order invariant of $F_{2}^{(k)}$, then $\psi_{k+1}=D_{1}^{(k+1)} \psi_{k}$ is a $(k+1)$ st invariant of $F_{2}^{(k+1)}$.

Proof. Suppose $\phi$ is a $k$ th order invariant of $F_{1}^{(k)}: X_{1}^{(k)} \phi=X_{3}^{(k)} \phi=0$. From this and the recurrence relations for $D_{i}^{(k)}, i=1,2$, equations (2.3), we easily deduce that $\partial_{u_{k+1,0}}\left(D_{2}^{(k+1)} \phi\right)=0$. So we have to show that $X_{1}^{(k+1)} D_{2}^{(k+1)} \phi=0$. Using Proposition 2.4, the recurrence relation for the total vector fields $D_{i}^{(k)}$, and the structure equations for $F^{(k+1)}$ we deduce that

$$
\begin{aligned}
X_{1}^{(k+1)} D_{2}^{(k+1)} \phi & =D_{1}^{(k+1)} D_{2}^{(k+1)} \phi+\left(D_{2}^{(k+1)}\right)^{k} f \partial_{u_{0, k+1}} D_{2}^{(k+1)} \phi \\
& =D_{1}^{(k+1)} D_{2}^{(k+1)} \phi+\left(D_{2}^{(k+1)}\right)^{k} f \partial_{u_{0, k}} \phi \\
& =D_{2}^{(k+1)} D_{1}^{(k+1)} \phi+\left(D_{1}^{(k+1)}\right)^{k} f \partial_{u_{k, 0}} \phi \\
& =D_{2}^{(k+1)} D_{1}^{(k+1)} \phi, \text { by definition of } \phi \\
& =D_{2}^{(k+1)}\left(X_{1}^{(k)}+u_{k+1,0} X_{3}^{(k)}\right) \phi=0 .
\end{aligned}
$$

A similar argument establishes that $D_{1}^{(k+1)} \psi$ is a $(k+1)$ st order invariant of $F_{2}^{(k+1)}$ if $\psi$ is a $k$ th order invariant of $F_{2}^{(k)}$. It is easy to see that $D_{2}^{(k+1)} \phi$ has order $k+1$. For, if it did not, then $\partial_{u_{0, k}} \phi=0$. But since $\partial_{u_{k, 0}} \phi=0$ this would imply that $\phi$ has order at most $k-1$, contrary to the definition of $\phi$. A similar argument holds for $D_{1}^{(k+1)} \psi$.

It is now not too difficult to show that if a partial differential equation (2.1) is Darboux integrable at order $k_{0}$, then the family $\omega_{k}=\psi_{k} d x+\phi_{k} d y, k \geq k_{0}$ of 1forms is an infinite number of nontrivial conservation laws for the equation. This in turn implies that every such equation possesses infinitely many linearizing Bianchi transformations. Note that an extensive study of conservation laws for second order hyperbolic partial differential equations has been carried out in [BGH] and in [AK]. In the latter reference it is shown, in particular, that every such Darboux integrable equation possesses infinitely many higher degree form-valued conservation laws. 
In order to provide further motivation for Definition 3.1, we conclude this section by noting one further important property of Darboux integrable equations. From the above discussion it is clearly possible to encounter equations where only one of the characteristic systems have two independent invariants at some order, say $k \geq 2$, while the other characteristic system has only one invariant. Such equations are called Darboux semi-integrable at order $k$. It turns out that in order for the Cauchy problem for a second order hyperbolic partial differential equation in one dependent and two independent variables to be solvable by ordinary differential equations, it is sufficient for the equation to be Darboux semi-integrable. For simplicity we will prove the result in the case of Darboux semi-integrability at order two, however, the result and its proof readily generalizes to all orders.

Proposition 3.3. Fix smooth real valued functions $\phi, \psi$ on a domain $W \subset \mathbb{R}^{2}$, smooth immersed curve $\gamma: I \subset \mathbb{R} \rightarrow W$ with a field $\mathbf{n}$ of unit normals along $\gamma$. Suppose the partial differential equation $u_{x y}=f\left(x, y, u, u_{x}, u_{y}\right)$ is Darboux semiintegrable at order 2 . Then

(i) The initial value problem

$$
\begin{gathered}
u_{x y}=f\left(x, y, u, u_{x}, u_{y}\right), \\
u=\phi, \quad \frac{\partial u}{\partial \mathbf{n}}=\psi, \quad \text { along } \gamma,
\end{gathered}
$$

has a unique smooth local solution whenever $\gamma$ does not have a tangent parallel to the $x$ or $y$ axes.

(ii) The solution arises as the flow of a unique smooth vector field determined by the initial conditions, the curve $\gamma$, the partial differential equation $u_{x y}=f$ and its characteristic invariants.

Proof. We will construct the vector field that provides the claimed solution. Since the equation is Darboux semi-integrable on the 2-jets, one at least of its characteristic systems $F_{1}^{(2)}, F_{2}^{(2)}$, has at least 2 invariants. Suppose that it is $F_{2}^{(2)}$. Consequently, the invariants include $x$; denote the highest order one by $I$. We can assume that $\partial_{u_{11}} I \neq 0$ for otherwise $I$ would be a first order invariant contradicting the hypothesis that the equation is semi-integrable at order 2. Fix any smooth real valued function $g: \mathbb{R} \rightarrow \mathbb{R}$ (the function $g$ is subsequently to be determined) and consider the submanifold $\mathcal{K}_{g}$ of the partial differential equation manifold $\mathcal{R}_{2}$ defined as the zero set of the invariant $I-g(x)$. By the implicit function theorem, we can express this zero set in the form $u_{11}=\lambda\left(x, y, u, u_{1}, u_{2} ; g(x)\right)$ for some smooth function $\lambda$ of the indicated arguments. The Vessiot distribution associated with the submanifold $\mathcal{K}_{g}$ has local basis

$$
\left\{K_{1}=\partial_{x}+u_{1} \partial_{u}+\lambda \partial_{u_{1}}+f \partial_{u_{2}}, K_{2}=\partial_{y}+u_{2} \partial_{u}+f \partial_{u_{1}}+u_{22} \partial_{u_{2}}, K_{3}=\partial_{u_{22}}\right\},
$$

with structure

$$
\left[K_{1}, K_{2}\right]=\left(K_{1} f-K_{2} \lambda\right) \partial_{u_{1}}-\left(K_{2} f\right) \partial_{u_{2}},\left[K_{1}, K_{3}\right]=0,\left[K_{3}, K_{2}\right]=\partial_{u_{2}}
$$

It is not difficult to show that $K_{1} f-K_{2} \lambda-0$ when it is taken into account that $u_{11}-\lambda=0$ is a level set of the invariant $I-g(x)$ of $F_{2}^{(2)}$. From this it is easy to see that distribution (3.2) has a 1-dimensional Cauchy subdistribution spanned by $Z=K_{1}+\left(K_{2} f\right) K_{3}$. That is, $\left[Z, K_{i}\right] \equiv 0 \bmod \left\{K_{1}, K_{2}, K_{3}\right\}, i=1,2,3$. Now we can "lift" the curve $\gamma$ in $W$ to a curve $\bar{\gamma}=\left(x(t), y(t), a(t), a_{1}(t), a_{2}(t)\right)$ in $J^{1}(W, \mathbb{R})$ 
by requiring that $\bar{\gamma}^{*}\left(d u-u_{1} d x-u_{2} d y\right)=0$. This implies that $\bar{\gamma}$ is a curve on the 1 -graph of some real valued function $v$ on $W$. This lift is not unique. However, if in addition $v$ is to satisfy the initial conditions in the statement of the proposition, then the lift is unique and given by $a(t)=(\phi \circ \gamma)(t)$ and where $a_{1}(t), a_{2}(t)$ satisfy

$$
\left(\begin{array}{cc}
\dot{x}(t) & \dot{y}(t) \\
n_{1}(t) & n_{2}(t)
\end{array}\right)\left(\begin{array}{l}
a_{1}(t) \\
a_{2}(t)
\end{array}\right)=\left(\begin{array}{c}
(\phi \circ \gamma)^{\prime}(t) \\
(\psi \circ \gamma)(t)
\end{array}\right)
$$

where $\mathbf{n}(t)=\left(n_{1}(t), n_{2}(t)\right)$. The curve $\bar{\gamma}$ so defined is a curve on the 1 -graph of some function $v$ satisfying the initial conditions involving $\phi$ and $\psi$. We now show that we can lift $\bar{\gamma}$ to a unique 1-dimensional integral submanifold of the contact structure of $J^{2}$ restricted to $\mathcal{H}_{g}$. That is, a curve $\tilde{\gamma}=\left(x(t), y(t), a(t), a_{1}(t), a_{2}(t), a_{22}(t)\right)$ satisfying $(\tilde{\gamma})^{*} i^{*} \Omega^{2}=0$, where $i: \mathcal{H}_{g} \rightarrow J^{2}$ is the inclusion. A short calculation shows that since $\gamma$ has tangents nowhere parallel to either the $x$ - or $y$-axes, this implies that the functions $g$ and $a_{22}$ are uniquely determined giving the claimed unique 1-dimensional integral submanifold of $i^{*} \Omega^{2}$. But we have already seen that distribution (3.2), $\left(i^{*} \Omega^{2}\right)^{\perp}$, has a 1-dimensional Cauchy distribution spanned by $Z$. The usual properties of Cauchy characteristics now guarantee that the 1-dimensional integral submanifold $\tilde{\gamma}$ is extended by $Z$ to a local 2-dimensional integral submanifold of $i^{*} \Omega^{2}$ since $Z$ is transverse to the initial data. This defines the 2-graph of a solution of the overdetermined system $u_{x y}=f, u_{x x}=\lambda$. The unique integral submanifold so constructed also satisfies the initial conditions. This proves both parts of the proposition.

Now when equation (2.1) is not merely Darboux semi-integrable but Darboux integrable, say, at order 2, then each characteristic system has a second order invariant. Suppose then that (2.1) has a second order invariant $J$ of $F_{1}^{(2)}$ in addition to invariant $I$ of $F_{2}^{(2)}$. Then for any fixed functions $g(x), h(y)$ we have the submanifold $\mathcal{K}_{g, h}$

$$
\begin{gathered}
u_{12}=f\left(x, y, u, u_{1}, u_{2}\right), \\
u_{11}=\lambda\left(x, y, u, u_{1}, u_{2} ; g(x)\right), \quad u_{22}=\mu\left(x, y, u, u_{1}, u_{2} ; h(y)\right)
\end{gathered}
$$

of $J^{2}$ and inclusion $i: \mathcal{K}_{g, h} \rightarrow J^{2}$, for some smooth functions $\lambda, \mu$, by the implicit function theorem. Then $\left(i^{*} \Omega^{2}\right)^{\perp}=\left\{K_{1}=\partial_{x}+u_{1} \partial_{u}+\lambda \partial_{u_{1}}+f \partial_{u_{2}}, K_{2}=\partial_{y}+\right.$ $\left.u_{2} \partial_{u}+f \partial_{u_{2}}+\mu \partial_{u_{2}}\right\}$ has structure $\left[K_{1}, K_{2}\right]=\left(K_{1} f-K_{2} \lambda\right) \partial_{u_{1}}+\left(K_{1} \mu-K_{2} f\right) \partial_{u_{2}}$. It is easy to show that, since $u_{11}=\lambda$ is a level set of the invariant $I-g(x)$ of $F_{2}^{(2)}$ and $u_{22}=\mu$ is a level set of the invariant $J-h(y)$ of $F_{1}^{(2)}$, then $K_{1}$ and $K_{2}$ commute. Thus, the Vessiot distribution $\left\{K_{1}, K_{2}\right\}$ for the overdetermined system (3.3) is Frobenius integrable and projects to the base $\mathbb{R}^{2}$. It follows from the proof of Proposition 2.2, that its leaves define 2-graphs of solutions of equations (3.3) and hence every pair of smooth functions $g, h$ furnishes smooth solutions of equation (2.1) and this by solving a given Frobenius system. So we conclude that if an equation (2.1) is Darboux integrable on the 2-jets, then smooth solutions depending upon two arbitrary smooth functions may be constructed by solving a Frobenius system, that is, by solving ordinary differential equations. This process of finding solutions of a partial differential equation is what was classically known as the "method of Darboux" and the solution depending upon two arbitrary functions that one obtained was called the general solution. This term is justified since the result just established has a converse, namely, every smooth solution of an equation (2.1) Darboux integrable on the 2-jets arises in precisely this way. To see this, fix a 
solution $u$ of (2.1) which is smooth on a domain $W$ of $\mathbb{R}^{2}$. Fix a smooth immersed curve $\gamma$ in $W$ with tangents not parallel to the $x$ - or $y$-axes. The function $u$ and curve $\gamma$ determine functions $\phi(t)$ and $\psi(t)$ by

$$
\phi(t)=(u \circ \gamma)(t), \quad \psi(t)=((\mathbf{n} . \nabla u) \circ \gamma)(t),
$$

and a unique curve $\bar{\gamma}$ in $J^{1}(W, \mathbb{R})$ by the equation $\bar{\gamma}^{*}\left(d u-u_{1} d x-u_{2} d y\right)=0$. If $\gamma(t)=(x(t), y(t))$ it is easy to see that $\bar{\gamma}$ is a 1-dimensional integral submanifold of $i^{*} \Omega^{2}=\left\{d u-u_{1} d x-u_{2} d y, d u_{1}-\lambda d x-f d y, d u_{2}-f d x-\mu d y\right\}$ for uniquely determined functions $g$ and $h$ since $\dot{x}(t) \dot{y}(t) \neq 0$. Denote the unique functions so determined by $\bar{g}, \bar{h}$. The corresponding Frobenius integrable Pfaff system $i^{*} \Omega^{2}$ determines a foliation of $\mathcal{K}_{\bar{g}, \bar{h}}$ by 2-dimensional leaves. The curve $\bar{\gamma}$ lies on exactly one leaf, $l_{0}$. By the proof of Proposition 2.2 these leaves define 2-graphs of solutions of the overdetermined system (3.3). Let $\bar{u}$ denote the solution of (3.3) defined by leaf $l_{0}$. Then by construction $\bar{u}$ satisfies $(\bar{u} \circ \gamma)(t)=\phi(t),((\mathbf{n} \cdot \nabla \bar{u}) \circ \gamma)(t)=\psi(t)$. But if equation (2.1) is Darboux integrable, then it is Darboux semi-integrable and the smooth existence and uniqueness result just established, Proposition 3.3. applies to show that in a neighborhood of the curve $\gamma, \bar{u}=u$, which is what we wanted to show. We can summarizes these calculations in the statement,

Proposition 3.4. An equation in $E$ is Darboux integrable on the 2-jets if and only if every smooth solution may be obtained from the solutions of a Frobenius system of the form $\left\{K_{1}, K_{2}\right\}$ for some (unique) smooth functions $g, h \square$

Of course similar results can be established for Darboux integrable semilinear equations on any jet. The restriction to the second jet is purely for simplicity of presentation.

Furthermore, restriction to the semilinear equations $E$ is also unnecessary. Similar results can be proved for fully nonlinear Darboux integrable and Darboux semiintegrable scalar second order partial differential equations in the plane. Indeed the fact that Darboux semi-integrable partial differential equations in this general case are solvable by ordinary differential equations was first proved by Goursat (see Go96]).

A key property that is claimed for Darboux integrable equations is that not only may the general solution be constructible from the solutions of a 2-parameter family of Frobenius systems, but, in addition, that solution is always expressible in finite terms of arbitrary functions and their derivatives. For the semilinear equations $E$ that are Darboux integrable on the 2-jets, Vessiot Vess39, Vess42 gave a beautiful geometric explanation for this. To briefly describe this, note that characteristic systems $F_{1}^{(2)}, F_{2}^{(2)}$ associated with say, a (2,2)-Darboux integrable equation, are each defined on 5-dimensional quotient manifolds obtained by factoring out the differential equations manifold $\mathcal{R}_{2}$ by the respective characteristic invariants. Thus each characteristic system is a rank 2 distribution on a 5 dimensional manifold 8 By explicit computation, Vessiot shows that each characteristic system on their respective quotient 5 -manifolds are diffeomorphic to the contact system on $J^{3}(\mathbb{R}, \mathbb{R})$.

\footnotetext{
${ }^{7}$ Note that these calculations also imply that when an equation is Darboux semi-integrable the construction of Frobenius integrable subdistributions of its Vessiot distribution in the manner of Proposition 2.2 may be carried out in the smooth as opposed to analytic category. However, as will by now be clear, this will not generally be possible without carrying out prolongations of the Vessiot distribution to an order higher than the order of the equation.

${ }^{8}$ Such systems are precisely the ones studied in general in Cartan [Ca].
} 
See also Va90 for a discussion of these remarks. Similar results arise in the case of equations $E$ which are (2,3)-Darboux integrable on the 2-jets.

Every Darboux integrable equation is semi-integrable. But at any fixed order there may be Darboux semi-integrable equations which are not Darboux integrable 9 The classification of these equations appears to be well beyond present capabilities. As we mentioned in \$1 Cartan's paper [Ca is devoted to a study of second order partial differential equations in one dependent and two independent variables which are Darboux semi-integrable at order 2 . As he shows, this involves the equivalence problem of three 1 -forms on a 5 -manifold. There seems little hope of making progress with this type of general classification at higher orders.

\section{TANGential CharaCteristic SYMmetries AND STRUCTURE OF MANIFOLDS OF HYPERBOLIC TYPE}

By contrast, I want to show that the problem of classifying the equations in $E$ that are Darboux integrable, though admittedly difficult, has significant possibilities; at least for gaining insight into the nature and structure of the moduli space for the problem.

To begin with, let's consider the Liouville equation

$$
u_{x y}+2 e^{u}=0 .
$$

It is not hard to show that it is $(2,2)$-Darboux integrable at order 2 and that $F_{1}^{(2)}$ has invariants $y$ and $\eta=\frac{1}{2}\left(\frac{1}{2} u_{y}^{2}-u_{y y}\right)$ and those of $F_{2}^{(2)}$ are $x$ and $\xi=\frac{1}{2}\left(\frac{1}{2} u_{x}^{2}-u_{x x}\right)$. An easy calculation shows that for each of $F_{1}^{(2)}$ and $F_{2}^{(2)}$, the Cauchy system is trivial. An infinitesimal symmetry of (say) $F_{2}^{(2)}$ which is tangent to the joint level sets of the invariants $x, y, \xi, \eta$ is a vector field, $V$, on the Liouville manifold, $\mathcal{R}$, such that $\left[V, X_{2}\right] \equiv 0,\left[V, X_{4}\right] \equiv 0, \bmod F_{2}^{(2)}, V(x, y, \xi, \eta)=(0,0,0,0)$. A computation reveals that the Lie algebra of these symmetries is finite dimensional with basis

$$
\begin{aligned}
& \bar{L}_{1}=\partial_{z}+p \partial_{p}+\partial^{2} \partial_{r}, \quad \bar{L}_{2}=-2 \partial_{p}-2 p \partial_{r}, \\
& \bar{L}_{3}=p \partial_{z}-2 e^{z} \partial_{q}+\frac{p^{2}}{2} \partial_{p}+\frac{p^{3}}{2} \partial_{r}-2 q e^{z} \partial_{t},
\end{aligned}
$$

and structure

$$
\left[\bar{L}_{1}, \bar{L}_{2}\right]=-\bar{L}_{2}, \quad\left[\bar{L}_{1}, \bar{L}_{3}\right]=\bar{L}_{3}, \quad\left[\bar{L}_{2}, \bar{L}_{3}\right]=-2 \bar{L}_{1} .
$$

Note that since we are here working on the 2-jets, we have used the Monge notation $z:=u, p:=u_{1,0}, q:=u_{0,1}, r:=u_{2,0}, t:=u_{0,2}$. A similar calculation for $F_{1}^{(2)}$ shows that these symmetries are spanned by

$$
\begin{aligned}
& \bar{R}_{1}=\partial_{z}+q \partial_{q}+q^{2} \partial_{t}, \quad \bar{R}_{3}=-2 \partial_{q}-2 q \partial_{t} \\
& \bar{R}_{2}=q \partial_{z}-2 e^{z} \partial_{p}+\frac{q^{2}}{2} \partial_{q}+\frac{q^{3}}{2} \partial_{t}-2 p e^{z} \partial_{r}
\end{aligned}
$$

with structure

$$
\left[\bar{R}_{1}, \bar{R}_{2}\right]=\bar{R}_{2}, \quad\left[\bar{R}_{1}, \bar{R}_{3}\right]=-\bar{R}_{3}, \quad\left[\bar{R}_{2}, \bar{R}_{3}\right]=2 \bar{R}_{1} .
$$

We observe that these Lie algebras are isomorphic to the simple complex Lie algebra $A_{1}$ and satisfy $\left[\bar{L}_{\alpha}, \bar{R}_{\beta}\right]=0$, for all $\alpha, \beta$. An easy calculation shows that they

\footnotetext{
${ }^{9}$ An example of an equation which is Darboux semi-integrable at every order but not Darboux integrable at any order is given by Juráš and Anderson in [JA].
} 
act simply transitively on the joint level sets of the 4 invariants $x, y, \xi, \eta$. Thus, we see that the infinitesimal symmetries of the characteristic systems $F_{1}^{(2)}$ and $F_{2}^{(2)}$ which are tangent to the joint level sets of the characteristic invariants are reciprocal, simply transitive Lie algebras of vectors fields of dimension 3 . Hence, by standard theory (see Sharpe [Sh], Chap. 3, Theorem 8.7) the Liouville differential equations manifold, $\mathcal{R}$, is foliated by 3 -dimensional submanifolds each of which is a local Lie group, locally isomorphic to $S L(2, \mathbb{C})$. In this paper we will refer to the symmetries of the characteristic systems $F_{1}^{(k)}$ and $F_{2}^{(k)}$ of a Darboux integrable equation that are tangent to the joint level sets of the characteristic invariants as tangential characteristic symmetries.

One can say a little more, since the vector fields $\left\{\partial_{x}, \partial_{y}, \partial_{r}, \partial_{t}, \bar{L}_{1}, \bar{L}_{2}, \bar{L}_{3}\right\}$ and $\left\{\partial_{x}, \partial_{y}, \partial_{r}, \partial_{t}, \bar{R}_{1}, \bar{R}_{2}, \bar{R}_{3}\right\}$ each form a frame field on the Liouville manifold, $\mathcal{R}$, we can express the Vessiot distribution in terms of these frame fields:

$$
F_{\text {Liouv. }}=\left\{\partial_{x}+\bar{L}_{3}+\xi \bar{L}_{2}, \partial_{r}\right\} \oplus\left\{\partial_{y}+\bar{R}_{2}+\eta \bar{R}_{3}, \partial_{t}\right\}
$$

as is easily verified. Thus, the Liouville equation determines a Lie group, $S L(2, \mathbb{C})$, and a hyperbolic structure expressed in terms of the left and right invariant vector fields on the group. A theorem of E. Vessiot [Vess39] Vess42] shows that these facts are general. That is, every semilinear partial differential equation (2,2)-Darboux integrable on the 2-jets is characterised by a complex Lie group of dimension 3 together with a canonical hyperbolic structure on a 7-dimensional manifold. This structure theorem accounts for most of the semilinear partial differential equations Darboux integrable on the 2-jets. As mentioned in $\$ 3$ the remaining ones are of two types: those that are $(2,3)$-Darboux integrable on the 2 -jets and those that are $(3,3)$-Darboux integrable on the 2-jets. The former case is defined by the contact orbit of equations 3.1a . Vessiot proves a similar structure theorem and shows that equations (3.1a) are 'associated' with the 2-dimensional complex Lie groups while in the latter he shows that every equation in $E$ which is $(3,3)$-Darboux integrable on the 2 -jets is contact equivalent to the linear wave equation $u_{x y}=0$. Vessiot shows that the 2-dimensional abelian group is associated with the linear equation in 3.1a while the nonlinear equation is associated with 2-dimensional nonabelian group. In view of Goursat's theorem that a second order scalar partial differential equation has at most three independent characteristic invariants on the 2 -jets, this exhausts the classification at that order.

Our aim in this section is first, to reveal the nature of this association between Lie group and Darboux integrable equation by showing that, as in the case of the Liouville equation, all the Lie groups in question arise precisely as the automorphisms of the characteristic systems which are tangent to the joint level sets of the characteristic invariants, the tangential characteristic symmetries. On aspect of this is that it enables us to 'see' the Lie groups that feature in Vessiot's structure theorem. Secondly, to show that all these facts admit generalization to all prolongations, showing that the classification problem for Lie groups is implied in the classification of Darboux integrable equations.

The first step is to study manifolds of $(p, q)$-hyperbolic type $\left(M, \mathcal{H}=\mathcal{H}_{1} \oplus \mathcal{H}_{2}\right)$ where $p=2$ and $q=2,3$. We refer to the hyperbolic structures as having the $(2,2)$ - or the $(2,3)$-configuration. It follows from our discussions in 92 and [3] that these manifolds are the basic geometric objects underlying that subset of $\operatorname{Darb}(E)$ where the equations are either $(2,2)$ - or $(2,3)$-Darboux integrable at some order. 
In this paper we have been able to obtain Vessiot-type structure theorems for only the manifolds of $(2,2)$ - and (2,3)-hyperbolic type. However, as we shall see this already implies the classification problem for Lie groups and establishes the point that the classification problem for the Darboux integrable semilinear equations contains the Lie group problem as a subproblem (among other things one has to decide which Lie groups can appear as tangential characteristic symmetries). We denote by $\operatorname{Aut}\left(\mathcal{H}_{i}\right)_{\text {tang. }}$ the (infinitesimal) tangential characteristic symmetries of the hyperbolic summands $\mathcal{H}_{i}$. We can now state the main structure theorem of this paper; it completely describes these symmetries.

Theorem 4.1a (Structure of manifolds of (2,2)-hyperbolic type). Let $\mathcal{H}=\mathcal{H}_{1} \oplus$ $\mathcal{H}_{2}$ be a $(2,2)$-hyperbolic structure on a $(n+4)$-dimensional manifold $M_{n+4}$. Let $\left(u, u_{0}\right)$ be two independent invariants of $\mathcal{H}_{2}$ and $\left(v, v_{0}\right)$ two independent invariants of $\mathcal{H}_{1}$. Then

a) The elements of $\operatorname{Aut}\left(\mathcal{H}_{1}\right)_{\text {tang. }}$ and $\operatorname{Aut}\left(\mathcal{H}_{2}\right)_{\text {tang. }}$ are, respectively, vector fields of the form

$$
\sum_{\alpha=1}^{n} \psi_{\alpha}\left(v, v_{0}\right) R_{\alpha} \quad \text { and } \quad \sum_{\alpha=1}^{n} \phi_{\alpha}\left(u, u_{0}\right) L_{\alpha},
$$

where $\mathcal{L}=\left\{L_{\alpha}\right\}_{\alpha=1}^{n}, \mathcal{R}=\left\{R_{\alpha}\right\}_{\alpha=1}^{n}$ are $n$-dimensional Lie algebras of vector fields tangent to and acting simply transitive on the joint level sets of the 4 invariants $u, u_{0}, v, v_{0}$, satisfying $[\mathcal{L}, \mathcal{R}]=0$. The functions $\phi, \psi$ are arbitrary.

b) There are local coordinates on $M$ such that the hyperbolic summands $\mathcal{H}_{1}, \mathcal{H}_{2}$ have local expression

$$
\mathcal{H}_{1}=\left\{\partial_{u}+L, \partial_{u_{0}}\right\}, \quad \mathcal{H}_{2}=\left\{\partial_{v}+R, \partial_{v_{0}}\right\},
$$

where $L \in \operatorname{Aut}\left(\mathcal{H}_{2}\right)_{\text {tang. }}, R \in \operatorname{Aut}\left(\mathcal{H}_{1}\right)_{\text {tang. }}$

Theorem 4.1b (Structure of manifolds of (2,3)-hyperbolic type). Let $\mathcal{H}=\mathcal{H} \oplus$ $\mathcal{H}_{2}$ be a $(2,3)$-hyperbolic structure on an $(n+5)$-dimensional manifold $M_{n+5}$. Let $\left(u, u_{0}, u_{1}\right)$ be three independent invariants of $\mathcal{H}_{2}$ and $\left(v, v_{0}\right)$ two independent invariants of $\mathcal{H}_{1}$. Then

a) The elements of $\operatorname{Aut}\left(\mathcal{H}_{1}\right)_{\text {tang. }}$ and $\operatorname{Aut}\left(\mathcal{H}_{2}\right)_{\text {tang. }}$ are, respectively, vector fields of the form

$$
\sum_{\alpha=1}^{n} \psi_{\alpha}\left(v, v_{0}\right) R_{\alpha} \quad \text { and } \quad \sum_{\alpha=1}^{n} \phi_{\alpha}\left(u, u_{0}, u_{1}\right) L_{\alpha}
$$

where $\mathcal{L}=\left\{L_{\alpha}\right\}_{\alpha=1}^{n}, \mathcal{R}=\left\{R_{\alpha}\right\}_{\alpha=1}^{n}$ are $n$-dimensional Lie algebras of vector fields tangent to and acting simply transitive on the joint level sets of the 4 invariants $u, u_{0}, u_{1}, v, v_{0}$, satisfying $[\mathcal{L}, \mathcal{R}]=0$. The functions $\phi, \psi$ are arbitrary.

b) There are local coordinates on $M$ such that the hyperbolic summands $\mathcal{H}_{1}, \mathcal{H}_{2}$ have local expression

$$
\mathcal{H}_{1}=\left\{\partial_{u}+u_{1} \partial_{u_{0}}+L, \partial_{U_{1}}\right\}, \quad \mathcal{H}_{2}=\left\{\partial_{v}+R, \partial_{v_{0}}\right\}
$$

where $L \in \operatorname{Aut}\left(\mathcal{H}_{2}\right)_{\text {tang. }}, R \in \operatorname{Aut}\left(\mathcal{H}_{1}\right)_{\text {tang. }}$.

Remark. Thus, according to Theorems 4.1a and 4.1b, $(2,2)$ - and (2,3)-hyperbolic structures have a local expression in terms of their symmetries. More precisely, a manifold of $(2,2)$ - or $(2,3)$-hyperbolic type of dimension $m$ has a foliation by 
(respectively) $(m-4)$-dimensional or $(m-5)$-dimensional leaves, the joint level sets of their invariants. Locally, each leaf has a framing in terms of the symmetries of the summands $\mathcal{H}_{1}$ or $\mathcal{H}_{2}$. Hence, every manifold of $(2,2)$ - or $(2,3)$-hyperbolic type of rank 4 and dimension $m$ is locally equivalent to either $\mathbb{R}^{4} \times G_{m-4}$ or to $\mathbb{R}^{5} \times G_{m-5}$, respectively, where $G_{m-4}$ and $G_{m-5}$ are Lie groups of dimension $m-4$ or $m-5$, respectively, $m \geq 6$. We shall refer to the coordinates determined by Theorems 4.1a and 4.1b as Vessiot normal coordinates.

Proof. The proof is adapted from Vessiot's arguments in Vess39. Let $\mathcal{H}=\mathcal{H}_{1} \oplus \mathcal{H}_{2}$ be a $(2,2)$-hyperbolic structure on $M_{n+4}$ with $\left(u, u_{0}\right)$ begin independent invariants of $\mathcal{H}_{2},\left(v, v_{0}\right)$ independent invariants of $\mathcal{H}_{1}$. We begin with an important preliminary general normal form result.

Lemma 4.2 (E. Vessiot). There are local coordinates $\left(u, u_{0}, v, v_{0}, x_{1}, x_{2}, \ldots, x_{n}\right)$ on $M_{n+4}$ such that

$$
\begin{aligned}
& \mathcal{H}_{1}=\left\{\partial_{u}+\sum_{i=1}^{n} A_{1}^{i} \partial_{x_{i}}, \partial_{u_{0}}+\sum_{i=1}^{n} A_{3}^{i} \partial_{x_{i}}\right\}, \\
& \mathcal{H}_{2}=\left\{\partial_{v}+\sum_{i=1}^{n} A_{2}^{i} \partial_{x_{i}}, \partial_{v_{0}}+\sum_{i=1}^{n} A_{4}^{i} \partial_{x}\right\},
\end{aligned}
$$

where $A_{1}^{i}, \ldots, A_{4}^{i}$ are smooth functions on $M_{n+4}$.

Proof. Fix an open set $V$ on $M_{n+4}$ with local coordinates that include the $\mathcal{H}_{2^{-}}$ invariants $u, u_{0}$. Hence, $\left(V ; u, u_{0}, y_{1}, \ldots, y_{n+2}\right)$ is a chart on $M_{n+4}$ for some collection of functions $y_{1}, \ldots, y_{n+2}$. Since $\left(v, v_{0}\right)$ are invariants for $\mathcal{H}_{1}$, every local basis $\left\{X_{1}, X_{3}\right\}$ of $\mathcal{H}_{1}$ in these coordinates has the form

$$
X_{1}=U_{1} \partial_{u}+U_{1}^{0} \partial_{u_{0}}+\tilde{A}_{1}^{i} \partial_{y_{i}}, \quad X_{3}=U_{3} \partial_{u}+U_{3}^{0} \partial_{u_{0}}+\tilde{A}_{3}^{i} \partial_{y_{i}},
$$

for some collection of smooth functions $U_{1}, \ldots, \tilde{A}_{3}^{i}$ on $M_{n+4}$. We show that in every such chart

$$
\operatorname{rank}(U)=\operatorname{rank}\left(\begin{array}{cc}
U_{1} & U_{1}^{0} \\
U_{3} & U_{3}^{0}
\end{array}\right)
$$

is 2 (possibly after shrinking neighborhoods). Suppose, on the contrary, that $\operatorname{rank}(U)$ is identically zero on $V$. Then $\mathcal{H}$ would have at least two invariants, $u$ and $u_{0}$, a contradiction. Suppose $\operatorname{rank}(U) \equiv 1$ on $V$. Then there is a change of basis so that $\mathcal{H}_{1}=\left\{\bar{X}_{1}=\partial_{u}+\omega \partial_{u_{0}}+A_{1}^{i} \partial_{y_{i}}, \bar{X}_{3}=A_{3}^{i} \partial_{y_{i}}\right\}$. From the structure equations for $\mathcal{H},\left[X_{2}, \bar{X}_{1}\right],\left[X_{4}, \bar{X}_{1}\right] \equiv 0 \bmod \mathcal{H}$. A short computation shows that, in consequence, $X_{2} \omega=X_{4} \omega=0$ in which case $\omega$ is an $\mathcal{H}_{2}$-invariant; thus $\omega=\phi\left(u, u_{0}\right)$ for some smooth $\phi$. This implies that any nonconstant solution $f\left(u, u_{0}\right)$ of

$$
\frac{\partial f}{\partial u}+\phi\left(u, u_{0}\right) \frac{\partial f}{\partial u_{0}}=0,
$$

provides a third independent invariant of $\mathcal{H}_{2}$ in addition to $\left(v, v_{0}\right)$, a contradiction. This establishes the claimed normal form for $\mathcal{H}_{1}$.

It is easy to see that $d u \wedge d u_{0} \wedge d v \wedge d v_{0} \neq 0$. For, suppose not. Then an equation of the form $G\left(u, u_{0}, v, v_{0}\right)=0$ must hold among the four invariants for some regular function $G$. By the implicit function theorem, we can solve for at least one of the two invariants $v$ or $v_{0}$ (for otherwise we have a contradiction of $d u \wedge d u_{0} \neq 0$ or $\left.d v \wedge d v_{0} \neq 0\right)$. Without loss of generality then we may suppose that there is a 
regular function, $h$, such that $v_{0}=h\left(u, u_{0}, v\right)$. Hence we have $0=d v_{0}\left(X_{1}\right)=$ $h_{u} d u\left(X_{1}\right)+h_{u_{0}} d u_{0}\left(X_{1}\right), 0=d v_{0}\left(X_{3}\right)=h_{u} d u\left(X_{3}\right)+h_{u_{0}} d u_{0}\left(X_{3}\right)$. This linear system for $h_{u}$ and $h_{u_{0}}$ has determinant $\left(d u \wedge d u_{0}\right)\left(X_{1}, X_{3}\right)$ which is not equal to zero by the arguments of the previous paragraph. But this implies that $v_{0}=h(v)$ which is not possible since $d v \wedge d v_{0} \neq 0$. This proves the claim. Taking a chart on $M_{n+4}$ of the form $\left(V ; u, u_{0}, v, v_{0}, x_{1}, x_{2}, \ldots, x_{n}\right)$ for some functions $x_{1}, x_{2}, \ldots, x_{n}$ and repeating the arguments of the first paragraph establishes the claimed normal forms for both $\mathcal{H}_{1}$ and $\mathcal{H}_{2}$.

From now on we assume that $\mathcal{H}_{1}$ and $\mathcal{H}_{2}$ are in the normal form provided by Lemma 4.2 It follows from the hyperbolic structure equations, $\left[\mathcal{H}_{1}, \mathcal{H}_{2}\right] \equiv 0 \bmod \mathcal{H}$, that $\left[X_{3}, X_{4}\right]=0$. Letting $u, v, x_{1}, x_{2}, \ldots, x_{n}$ be a complete set of local invariants of the Frobenius integrable distribution $\left\{X_{3}, X_{4}\right\}$ leads to the local basis

$$
\begin{aligned}
& \mathcal{H}_{1}=\left\{\partial_{u}+\lambda_{\alpha}\left(u, u_{0}, v, v_{0}, x\right) \partial_{x_{\alpha}}, \partial_{u_{0}}\right\}=\left\{X_{1}, X_{3}\right\}, \\
& \mathcal{H}_{2}=\left\{\partial_{v}+\rho_{\alpha}\left(u, u_{0}, v, v_{0}, x\right) \partial_{x_{\alpha}}, \partial_{v_{0}}\right\}=\left\{X_{2}, X_{4}\right\},
\end{aligned}
$$

for some set of smooth functions $\lambda_{\alpha}, \rho_{\alpha}$. One deduces easily that the $\lambda_{\alpha}$ do not depend on $v_{0}$ and the $\rho_{\alpha}$ do not depend upon $u_{0}$. From the structure equations we have $\left[X_{1}, X_{2}\right]=\left[X_{1}, X_{4}\right]=0$ and from the local expressions for $\mathcal{H}_{1}$ and $\mathcal{H}_{2}$ we have

$$
\left[\partial_{u_{0}},\left[X_{1}, X_{j}\right]\right]=\left[L_{u_{0}}^{\prime}, X_{j}\right]=0, \quad j=2,4
$$

where

$$
L=\lambda_{\alpha} \partial_{x^{\alpha}}, \quad L_{u_{0}}^{\prime}=\frac{\partial \lambda_{\alpha}}{\partial u_{0}} \partial_{x^{\alpha}} .
$$

We will continue to use the summation convention whenever there is no possibility of confusion. Otherwise an explicit sum sign will be used. We will also use the notation

$$
L_{y}^{(r)}=\frac{\partial^{r} \lambda^{\alpha}}{\partial y^{r}} \partial_{x^{\alpha}} .
$$

Despite its specialized appearance, the following lemma (which we shall use repeatedly) and the hyperbolic structure equations are mostly responsible for Theorems $4.1 \mathrm{a}$ and $4.1 \mathrm{~b}$.

Lemma 4.3. Let $\mathcal{D}$ be a distribution with local basis $X_{1}, X_{2}, \ldots, X_{l}$ on an $m$ dimensional manifold $M$ such that

1) $\mathcal{D}$ has a complete set of local invariants $y, z_{1}, \ldots, z_{a}$.

2) There are functions $w_{1}, \ldots, w_{b}$ on $M$ such that $y, z, w$ are functionally independent and $a+b+1<m$.

3) $\left[\partial_{y}, X_{i}\right]=0, i=1,2, \ldots, l$.

Let a nonzero vector field $L$ on $M$ be tangent to the joint level sets of the functions $y, z, w$, denote by $M / \sim$ and satisfying

$$
\left[L_{y}^{(r)}, X_{i}\right]=0, \quad i=1,2, \ldots, l,
$$

for some $r \geq 0$. Then if $r=0$ there are functions $\theta_{1}(y, z), \theta_{2}(y, z), \ldots, \theta_{q}(y, z)$ which, for each $z$ are linearly independent functions of $y$ (y-linearly independent) 
and vector fields $L_{1}, L_{2}, \ldots, L_{q}$ tangent to $M / \sim$, such that

$$
L=\sum_{\alpha=1}^{q} \theta_{\alpha}(y, z) L_{\alpha}, \quad L_{\alpha}=\sum_{\alpha=1}^{q} \lambda_{\alpha \beta}(z, w, x) \partial_{x_{\beta}},
$$

where $x_{1}, \ldots, x_{q}$ are local coordinates on $M / \sim, a+b+q+1=m$. If $r>0$, there are $y$-linearly independent functions $1, y, y^{2}, \ldots, y^{r-2}, \theta_{r}(y, z), \ldots, \theta_{q+r-1}(y, z)$ and vector fields $L_{\alpha}, \alpha=0,1, \ldots, q+r-1$, such that

$$
L=L_{0}+y L_{1}+\cdots+y^{r-1} L_{r-1}+\sum_{\alpha=r}^{q+r-1} \theta_{\alpha}(y, z) L_{\alpha} .
$$

Before proving Lemma 4.3, we apply it to the vector field $L=\lambda^{\alpha}\left(u, v, u_{0}, x\right) \partial_{x^{\alpha}}$ which satisfies equations (4.1). We take for $M$, the manifold $M_{n+4}$ and for $\mathcal{D}$ the distribution $\mathcal{H}_{2}$. The vector field $L$ is tangent to the joint level sets of the invariants $u, u_{0}, v, v_{0}$ and hence in particular to the joint level sets of the $\mathcal{H}_{2}$-invariants $u, u_{0}$. Noting that equations (4.1) have the form (4.2) with $r=1$ and $u_{0}$ playing the role of $y$, we conclude that there are vector fields $L_{\beta}=\lambda_{\beta}^{\alpha}(u, v, x) \partial_{x^{\alpha}}, \beta=0,1,2, \ldots, n$, tangent to the joint level sets of the four invariants and $u_{0}$-linearly independent functions $1, \phi_{1}\left(u, u_{0}\right), \ldots, \phi_{n}\left(u, u_{0}\right)$ such that

$$
L=L_{0}+\sum_{\beta=1}^{n} \phi_{\beta}\left(u, u_{0}\right) L_{\beta} .
$$

We note that in this instance $a=1\left(z_{1}:=u\right), b=2\left(w_{1}:=v, w_{2}:=v_{0}\right), m=$ $n+4, q=m-a-b-1=n$. The local expression for $\mathcal{H}_{1}$ now reads

$$
\mathcal{H}_{1}=\left\{\partial_{u}+L_{0}+\sum_{\alpha=1}^{n} \phi_{\alpha}\left(u, u_{0}\right) L_{\alpha}, \partial_{U_{0}}\right\} \text {. }
$$

Proof of Lemma 4.3. Look at case $r=0$. We have $\left[X_{i}, L\right]=\left[X_{i}, \partial_{y}\right]=0$; it follows from the Jacobi identity that $\left[X_{i}, L_{y}^{\prime}\right]=0$, for all $i$. Continuing to use the Jacobi identity shows that for all $i$ and $p \geq 0,\left[X_{i}, L_{y}^{(p)}\right]=0$. Among the sets

$$
\Lambda_{s}=\left\{L, L_{y}^{\prime}, L_{y}^{\prime \prime}, \ldots, L_{y}^{(s)}\right\}, \quad 0 \leq s \leq q-1,
$$

there is a smallest $s$ such that $\Lambda_{s}$ and $\Lambda_{s+1}$ have the same dimension $s+1$. After a little argument one sees that there are functions $\phi_{0}, \phi_{1}, \ldots, \phi_{s}$ on $M$ such that

$$
L_{y}^{(s+1)}=\phi_{0} L+\phi_{1} L_{y}^{\prime}+\cdots+\phi_{s} L_{y}^{(s)} .
$$

Using the fact that each $X_{i}$ commutes with the $L_{y}^{(p)}$ and the linear independence of the set $\Lambda_{s}$ it follows that the $\phi$ 's only depend upon the invariants of distribution $\mathcal{D}$, that is, $\phi_{j}=\phi_{j}(y, z)$. From the last displayed equation we have that the coefficients of $L, \lambda_{\alpha}$, satisfy a parametrized family of linear ordinary differential equations

$$
\frac{\partial^{s+1} \lambda_{\alpha}}{\partial y^{s+1}}=\phi_{0}(y, z) \lambda_{\alpha}+\cdots+\phi_{s}(y, z) \frac{\partial^{s} \lambda_{\alpha}}{\partial y^{s}} \text {. }
$$

If $s<q-1$ we may differentiate the last equation with respect to $y$ to obtain an equation of the form

$$
\frac{\partial^{q} \lambda_{\alpha}}{\partial y^{q}}=\phi_{0}(y, z) \lambda_{\alpha}+\cdots+\phi_{q-1}(y, z) \frac{\partial^{q-1} \lambda_{\alpha}}{\partial y^{q-1}} .
$$


That is, a parametrized family of $q$ th-order linear ordinary differential equations. The parameter is $z$. For each $z$, let $\theta_{1}(y, z), \theta_{2}(y, z), \ldots, \theta_{q}(y, z)$ be a fundamental set. It follows that the coefficients of $L$ have the form

$$
\lambda_{\alpha}(y, z, w, x)=\sum_{\beta=1}^{q} \lambda_{\alpha \beta}(z, w, x) \theta_{\beta}(y, z),
$$

for some collection of functions $\lambda_{\alpha \beta}$. Hence

$$
L=\sum_{\alpha=1}^{q} \theta_{\alpha}(y, z) L_{\alpha}, \quad L_{\alpha}=\sum_{\beta=1}^{q} \lambda_{\alpha \beta}(z, w, x) \partial_{x^{\beta}}
$$

as claimed. The proof for $r>0$ is similar.

As we did for $\mathcal{H}_{1}$, we may apply Lemma 4.3 to show that there are $v_{0}$-linearly independent functions $1, \psi_{1}\left(v, v_{0}\right), \ldots, \psi_{n}\left(v, v_{0}\right)$ and vector fields $R_{0}, R_{1}, \ldots, R_{n}$ tangent to the joint level sets of the invariants $u, u_{0}, v, v_{0}, M_{n+4} / \sim$, such that $\mathcal{H}_{2}$ has local expression

$$
\mathcal{H}_{2}=\left\{\partial_{v}+R_{0}+\sum_{\alpha=1}^{n} \psi_{\alpha}\left(v, v_{0}\right) R_{\alpha}, \partial_{v_{0}}\right\}
$$

From the equation $\left[X_{1}, X_{2}\right]=0$ we easily deduce that the vector fields $\partial_{u}+L_{0}$ and $\partial_{v}+R_{0}$ commute and introducing as new coordinates their $n+2$ independent joint invariants $u_{0}, v_{0}, w_{1}(u, v, x), \ldots, w_{n}(u, v, x)$ transforms $\mathcal{H}_{1}, \mathcal{H}_{2}$ to

$$
\begin{aligned}
& \mathcal{H}_{1}=\left\{\partial_{u}+\sum_{\alpha=1}^{n} \phi_{\alpha}\left(u, u_{0}\right) L_{\alpha}, \partial_{u_{0}}\right\}=\left\{X_{1}, X_{3}\right\}, \\
& \mathcal{H}_{2}=\left\{\partial_{v}+\sum_{\alpha=1}^{n} \psi_{\alpha}\left(v, v_{0}\right) R_{\alpha}, \partial_{v_{0}}\right\}=\left\{X_{2}, X_{4}\right\},
\end{aligned}
$$

where $L_{\alpha}=\lambda_{\alpha \beta}(u, v, w) \partial_{w_{\beta}}$, after redefining $\lambda_{\alpha \beta}$ and $R_{\alpha}=\rho_{\alpha \beta}(u, v, w) \partial_{w_{\beta}}$ after redefined $\rho_{\alpha \beta}$. We have

$$
0=\left[X_{1}, X_{2}\right]=\left[\partial_{u}, X_{2}\right]+\phi_{a}\left(u, u_{0}\right)\left[L_{\alpha}, X_{2}\right],
$$

and by the linear independence of the set $\left\{1, \phi_{1}, \ldots, \phi_{n}\right\}$ we obtain the equations

$$
\left[\partial_{u}, X_{2}\right]=\left[L_{\alpha}, X_{2}\right]=0
$$

showing that the $\rho_{\alpha \beta}$ are independent of $u$. By a similar calculation, making use of the local expression for $X_{2}$ we deduce that the $\lambda_{\alpha \beta}$ are independent of $v$ together with the equations $\left[R_{\alpha}, X_{1}\right]=0$ from which we deduce that

$$
\left[L_{\alpha}, R_{\beta}\right]=0, \quad \text { for all } \alpha, \beta=1,2, \ldots, n,
$$

upon using the independence of the $\phi$ 's and $\psi$ 's. We may now apply Lemma 4.3 to the equations (4.4) to deduce that for each $\alpha$ there are linearly independent functions $\sigma_{\gamma}^{\alpha}(v), \gamma=1,2, \ldots, n$, such that

$$
\rho_{\alpha \beta}(v, w)=\sum_{\gamma=1} \sigma_{\gamma}^{\alpha}(v) \rho_{\alpha \beta}^{\gamma}(\omega)
$$


for some collection of functions $\rho_{\alpha \beta}^{\gamma}$, that only depend upon $w$. Here, $\left\{L_{\alpha}\right\}_{\alpha=1}^{n}$ plays the role of distribution $\mathcal{D}$ in Lemma 4.3 and $v$ plays the role of $y$; parameter $r=0$. Setting

$$
R_{\alpha}^{\gamma}=\sum_{\beta=1}^{n} \rho_{\alpha \beta}^{\gamma}(w) \partial_{w^{\beta}},
$$

we have

$$
R_{\alpha}=\sum_{\gamma=1}^{n} \sigma_{\gamma}^{\alpha}(v) R_{\alpha}^{\gamma} .
$$

Reversing the role of $\left\{L_{\alpha}\right\}$ and $\left\{R_{\alpha}\right\}$ in the application of Lemma 4.3 to equations (4.4) and letting $u$ play the role of $y$, we see that for each $\alpha$, there are linearly independent functions $\tau_{\gamma}^{\alpha}(u), \gamma=1,2, \ldots, n$, and vector fields $L_{\alpha}^{\gamma}$ tangent to $M_{n+4} / \sim$ such that

$$
L_{\alpha}=\sum_{\gamma=1}^{n} \tau_{\gamma}^{\alpha}(u) L_{\alpha}^{\gamma} .
$$

where the coefficients of the $L_{\alpha}^{\gamma}$ only depend upon $w$.

Now an infinitesimal symmetry of the distribution $\mathcal{H}_{1}$ which, in addition, is tangent to $M_{n+4} / \sim$ is a vector field $V$ on $M_{n+4}$ satisfying $\left[V, X_{1}\right]=\left[V, X_{3}\right]=0$ and $V\left(u, u_{0}, v, v_{0}\right)=(0,0,0,0)$. From the local expression for $\mathcal{H}_{1}$ given above, it follows that $V$ has the form

$$
V=\sum_{\alpha=1}^{n} V^{\alpha}\left(v, v_{0}, w\right) \partial_{w^{\alpha}},
$$

and for each $\beta$ satisfies the equations

$$
\left[L_{\beta}, V\right]=0 .
$$

Systematically applying Lemma 4.3 to (4.5) taking for $\mathcal{D}$ the distribution $\left\{L_{\alpha}\right\}$ and for $y$ the variable $v_{0}$, shows that there are $v_{0}$-linearly independent functions $\eta_{\gamma}\left(v, v_{0}\right)$ and vector fields $\Delta_{\gamma}=\Delta_{\gamma}^{\alpha}(v, w) \partial_{w^{\alpha}}$ such that

$$
V=\sum_{\gamma=1}^{n} \eta_{\gamma}\left(v, v_{0}\right) \Delta_{\gamma} .
$$

Here, again, the parameter $r$ is zero. It follows from this, equations (4.5) and the linear independence of the $\eta_{\gamma}$ and $\tau_{\gamma}^{\beta}$ that for each $\alpha, \beta, \gamma$, we have

$$
\left[\Delta_{\alpha}, L_{\beta}^{\gamma}\right]=0
$$

Once again applying Lemma 4.3 to equations (4.6) shows that there are linearly independent functions $\delta_{\sigma}(v)$ and vectors fields $F_{\gamma}^{\sigma}=f_{\gamma}^{\alpha \sigma}(w) \partial_{w^{\alpha}}$ such that

$$
\Delta_{\gamma}=\sum_{\sigma=1}^{n} \delta_{\sigma}(v) F_{\gamma}^{\sigma} .
$$

Combining this with the expression for $V$ shows that there are functions $\eta_{\sigma \gamma}\left(v, v_{0}\right)$ such that

$$
V=\sum_{\sigma, \gamma=1}^{n} \eta_{\sigma \gamma}\left(v, v_{0}\right) F_{\gamma}^{\sigma}
$$


We have therefore shown that every infinitesimal symmetry of $H_{1}$ which is tangent to the joint level sets $M_{n+4} / \sim$ has the form (4.7).

Now among the $L_{\beta}^{\gamma}$, at most $n$ of them are linearly independent, say a maximal set $\bar{L}_{1}, \bar{L}_{2}, \ldots, \bar{L}_{q_{1}}, 0<q_{1} \leq n$. Note that if $q_{1}$ were zero we would have a contradiction of the hyperbolic structure equations. So there are functions $l_{\beta}^{\gamma j}(w)$ such that

$$
L_{\beta}^{\gamma}=\sum_{j=1}^{q_{1}} l_{\beta}^{\gamma j}(w) \bar{L}_{j}
$$

Since each vector field $R_{\alpha}^{\gamma}$ commutes with each $L_{\beta}^{\gamma}$, we find that the $l_{\beta}^{\gamma j}(w)$ are invariants of the distribution $\left\{R_{\alpha}^{\gamma}\right\}_{\alpha, \gamma=1}^{n}$ and these are necessarily constants (for otherwise distribution $\mathcal{H}_{2}$ would have independent invariants beyond $u, u_{0}$ ). By a similar argument there are functions $r_{\alpha}^{\gamma i}(w)$ such that

$$
R_{\alpha}^{\gamma}=\sum_{i=1}^{q_{2}} r_{\alpha}^{\gamma i}(w) \bar{R}_{i}
$$

for some maximal independent set $\left\{\bar{R}_{i}\right\}_{i=1}^{q_{2}}, 0<q_{2} \leq n$, of the $R_{\alpha}^{\gamma}$. It follows immediately, after making some obvious substitutions that there are functions $\bar{\phi}_{j}\left(u, u_{0}\right)$ such that

$$
X_{1}=\partial_{u}+\sum_{j=1}^{q_{1}} \bar{\phi}_{j}\left(u, u_{0}\right) \bar{L}_{j}
$$

Suppose that the number of linearly independent $\bar{L}_{j}$ appearing in the expression for $X_{1}$ is $a_{1}$, where $0<a_{1} \leq q_{1} \leq n$. Once again $a_{1}$ must be at least one in conformity with the structure equations of Definition 2.10. Without loss of generality we may take the set of functions $\left\{\phi_{1}, \ldots, \phi_{a_{1}}\right\}$ to be linearly independent for otherwise the number of linearly independent $\bar{L}_{j}$ may be reduced below $a_{1}$. But, if $a_{1}<n$, then it cannot be that the distribution $\mathcal{A}=\left\{\bar{L}_{1}, \ldots, \bar{L}_{a_{1}}\right\}$ is Frobenius integrable for this would define new independent invariants of the distribution $\mathcal{H}_{1}$. Indeed it is obvious that the flag of derived distributions of $\mathcal{A}$ must stabilize in dimension $n$. Denote by $\mathcal{L}=\left\{L_{1}, L_{2}, \ldots, L_{n}\right\}$ the last term in the derived flag of $\mathcal{A}$. Call this the completion of $\mathcal{A}$. We have now from the structure equations $\left[\mathcal{H}_{1}, \mathcal{H}_{2}\right]=0$, the equations $\left[X_{2}, X_{1}\right]=\left[X_{4}, X_{1}\right]=0$ and it follows that for each vector field in $\mathcal{A}$, we have

$$
\left[X_{2}, L_{j}\right]=\left[X_{4}, L_{j}\right]=0, \quad j=1,2, \ldots, a_{1} .
$$

From the Jacobi identity we easily deduce that

$$
\left[X_{2},\left[L_{\alpha}, L_{\beta}\right]\right]=\left[X_{4},\left[L_{\alpha}, L_{\beta}\right]\right]=0 .
$$

If $c_{\alpha \beta}^{\gamma}(w)$ is a set of functions expressing the relationship

$$
\left[L_{\alpha}, L_{\beta}\right]=c_{\alpha \beta}^{\gamma} L_{\gamma},
$$

between the elements of $\mathcal{L}$, we deduce from (4.8) that the $c_{\alpha \beta}^{\gamma}(w)$ are invariants of distribution $\mathcal{H}_{2}$ and must therefore be constants. All this leads us to conclude that the distribution $\mathcal{L}$ defines a simply transitive Lie algebra of vector fields tangent to $M_{n+4} / \sim$.

Now we may argue in a completely analogous fashion to establish the existence of an $a_{2}$-dimensional distribution $\mathcal{B}=\left\{R_{1}, R_{2}, \ldots, R_{a_{2}}\right\}, 0<a_{2} \leq q_{2} \leq n$, on 
$M_{n+4} / \sim$ featured in the local expression for $X_{2}$. It is easy to see that this distribution must commute with $\mathcal{A}$ and its derived flag must stabilize in dimension $n$. By the Jacobi identity, its completion must commute with $\mathcal{L}$. It follows from this that $\mathcal{B}$ determines a simply transitive Lie algebra of vector fields $\mathcal{R}$, the completion of $\mathcal{B}$, tangent to $M_{n+4} / \sim$. Hence we have shown that the hyperbolic structure $\mathcal{H}$ on $M_{n+4}$ determines a pair of reciprocal simply transitive Lie algebras of vector fields on $M_{n+4} / \sim$. That is, simply transitive Lie algebras $\mathcal{L}$ and $\mathcal{R}$ satisfying $[\mathcal{L}, \mathcal{R}]=0$. By standard theory (see Sharpe $[\mathrm{Sh}]$, Chapter 3, Theorem 8.7) this means that each joint level set of the four independent invariants $u, u_{0}, v, v_{0}$ has the structure of a local Lie group of dimension $n \geq 2$. So every manifold of $(2,2)$-hyperbolic type determines a Lie group of dimension at least 2. But what is this Lie group?

Recall we had earlier shown that the infinitesimal symmetries of the summand $\mathcal{H}_{1}$, which in addition are tangent to $M_{n+4} / \sim$, have the form (4.7). In addition we have seen that there is a Lie algebra of vector fields, $\mathcal{R}$, tangent to $M_{n+4} / \sim$ satisfying $\left[\mathcal{R}, X_{1}\right]=\left[\mathcal{R}, X_{3}\right]=0$. Consequently, $\mathcal{R}$ is a subalgebra of the Lie algebra of tangential characteristic symmetries of $\mathcal{H}_{1}$. But it is now easy to see that in fact $\mathcal{R}$ spans the entire Lie algebra of such symmetries. Indeed, from the form of the vector field $X_{1}$, we see that any tangential characteristic symmetry $V$ of $\mathcal{H}_{1}$ must commute with each element of $\mathcal{A}$ and hence with its completion $\mathcal{L}$. From the form of symmetry $V$, after taking into account the linear independence of the functions $\eta_{\gamma}\left(v, v_{0}\right)$ and $\delta_{\sigma}(v)$, defined earlier, and using equation (4.6), we see that $\left[L_{j}, F_{\gamma}^{\sigma}\right]=0, j=1,2, \ldots, a_{1} ; \sigma, \gamma=1,2, \ldots, n$. Since the distribution $\mathcal{R}$ locally defines a frame field on each joint level set, it follows that there are functions $h_{\gamma}^{\sigma \nu}(w)$ such that

$$
F_{\gamma}^{\sigma}=\sum_{\nu=1}^{n} h_{\gamma}^{\sigma \nu}(w) R_{\nu}
$$

We now easily deduce that the functions $h_{\gamma}^{\sigma \nu}(w)$ are invariants of $\mathcal{L}$ and must therefore be constants. Hence each $F_{\gamma}^{\sigma}$ is an element of the Lie algebra $\mathcal{R}$. By a similar argument one finds that every tangential characteristic symmetry $W$ of $\mathcal{H}_{2}$ has the form

$$
W=\sum_{\gamma=1}^{n} \omega_{\gamma}\left(u, u_{0}\right) L_{\gamma}
$$

for some choice of functions $\omega_{\gamma}\left(u, u_{0}\right)$ where the $L_{\gamma}$ form a basis for $\mathcal{L}$. This concludes the proof of Theorem 4.1a

We now turn to the manifolds (2,3)-hyperbolic type. Let $\mathcal{H}=\mathcal{H}_{1} \oplus \mathcal{H}_{2}$ be a $(2,3)$ hyperbolic structure on an $n+5$-dimensional manifold $M_{n+5}, n \geq 1$. Let $u, u_{0}, u_{1}$ denote the independent invariants of $\mathcal{H}_{2}$ and $v, v_{0}$ the independent invariants of $\mathcal{H}_{1}$. There is a local normal form result analogous to Lemma 4.2 and whose proof is similar.

Lemma 4.4 (E. Vessiot). If $\mathcal{H}=\mathcal{H}_{1} \oplus \mathcal{H}_{2}$ is a $(2,3)$-hyperbolic structure on $M_{n+5}$, there are local coordinates $\left(u, u_{0}, u_{1}, v, v_{0}, x_{1}, x_{2}, \ldots, x_{n}\right)$ such that 


$$
\begin{gathered}
\mathcal{H}_{1}=\left\{\partial_{u}+u_{1} \partial_{u_{0}}+\sum_{i=1}^{n} A_{1}^{i} \partial_{x_{i}}, \partial_{u_{1}}+\sum_{i=1}^{n} A_{3}^{i} \partial_{x_{i}}\right\}, \\
\mathcal{H}_{2}=\left\{\partial_{v}+\sum_{i=1}^{n} A_{2}^{i} \partial_{x_{i}}, \partial_{v_{0}}+\sum_{i=1}^{n} A_{4}^{i} \partial_{x_{i}}\right\}
\end{gathered}
$$

where $A_{1}^{i}, \ldots, A_{4}^{i}$ are smooth functions on $M_{n+5}$.

Theorem $4.1 \mathrm{~b}$ is proved in a manner analogous to Theorem $4.1 \mathrm{a}$ beginning with Lemma 4.4 instead of Lemma 4.2 ,

We may now apply Theorem 4.1 to the analysis of the space of $(2,2)$ - and $(2,3)$ Darboux integrable equations in the equation class $E$. For by Proposition 2.5, each such equation has a differential equations manifold which is a manifold of either $(2,2)$ - or a (2,3)-hyperbolic type of rank 4 and dimension $2 k+3$.

Theorem 4.5. Let $k$ be an integer greater than or equal to 2. Every partial differential equation in $E$ which is $(2,2)$-Darboux integrable on the $k$-jets has a Lie group of tangential characteristic symmetries of dimension $2 k-1$. Every partial differential equation in $E$ which is $(2,3)$-Darboux integrable on the $k$-jets has a Lie group of tangential characteristic symmetries of dimension $2 k-2$.

\section{Some illustrative EXAmples}

In this section we give a number of examples to illustrate Theorem $4.1 \mathrm{a}$

Example 5.1. The tangential characteristic symmetries of the Liouville equation given at the beginning of \$₫l may be placed the normal form guaranteed by Theorem $4.1 \mathrm{a}$ by the transformation

$$
\begin{aligned}
\sigma:(x, y, z, p, q, r, t) & \mapsto\left(x, y, e^{z}-p q / 4,-p / 2,-q / 2,\left(p^{2} / 2-r\right) / 2,\left(q^{2} / 2-t\right) / 2\right) \\
& =\left(u, v, w_{1}, w_{2}, w_{3}, u_{0}, v_{0}\right) .
\end{aligned}
$$

The corresponding canonical hyperbolic structure for the Liouville equation then has the form

$$
H_{\text {Liouv. }}=\left\{\partial_{u}+L_{3}+u_{0} L_{2}, \partial_{u_{0}}\right\} \oplus\left\{\partial_{v}+R_{2}+v_{0} R_{3}, \partial_{v_{0}}\right\},
$$

where

$$
\begin{gathered}
\sigma_{*}\left(\bar{L}_{1}\right)=L_{1}=w_{1} \partial_{w_{1}}+w_{2} \partial_{w_{2}}, \sigma_{*}\left(\bar{L}_{2}\right)=L_{2}=w_{3} \partial_{w_{1}}+\partial_{w_{2}}, \\
\sigma_{*}\left(\bar{L}_{3}\right)=L_{3}=w_{1} \partial_{w_{3}}-w_{2} w_{\alpha} \partial_{w_{\alpha}},
\end{gathered}
$$

and

$$
\begin{gathered}
\sigma_{*}\left(\bar{R}_{1}\right)=R_{1}=w_{1} \partial_{w_{1}}+w_{3} \partial_{w_{3}}, \quad \sigma_{*}\left(\bar{R}_{3}\right)=R_{3}=w_{2} \partial_{w_{1}}+\partial_{w_{3}}, \\
\sigma_{*}\left(\bar{R}_{2}\right)=R_{2}-w_{1} \partial_{w_{2}}-w_{3} w_{\alpha} \partial_{w_{\alpha}} .
\end{gathered}
$$

The reciprocal simply transitive Lie algebras of vector fields $\mathcal{L}=\left\{L_{\alpha}\right\}_{\alpha=1}^{3}, \mathcal{R}=$ $\left\{R_{\alpha}\right\}_{\alpha=1}^{3}$ are isomorphic to the left and right invariant vector fields on $S L(2, \mathbb{C})$. Note that in his treatment of the Liouville equation in Vess42, Vessiot begins with these Lie algebras and then (somewhat mysteriously, it seems) writes down the transformation $\sigma$ showing that this Lie group and hyperbolic structure is associated with the Liouville equation. In any case, a construction of the transformations that map a hyperbolic structure in Vessiot normal coordinates to the structure in jet bundle coordinates is given in Va94. In particular, this enables one to derive 
the transformation $\sigma$ above. More generally, it enables one, given a hyperbolic structure on a 7-dimensional manifold in Vessiot normal coordinates, to construct the associated Darboux integrable equation. In this paper we have shown how to proceed in the other direction: given an equation in $E$ either $(2,2)$ - or $(2,3)$ Darboux integrable on the $k$-jets, $k \geq 2$, to identify the Lie group in Vessiot's structure theorem. Thus, we can 'see' the Lie group buried in the canonical Vessiot distributions.

Example 5.2. The equation

$$
u_{x y}+2 \frac{\sqrt{u_{x} u_{y}}}{x+y}=0
$$

occurs in Goursat's original classification Go99. It is (2,2)-Darboux integrable on the 2-jets as may be shown by direct computation. Vessiot [Vess42, pp. 57-58] shows that it is associated with the nilpotent Lie group $G_{3}$ isomorphic to the subgroup $(x, y) \mapsto\left(x+a_{3} y+a_{1}, y+a_{2}\right)$ of the affine transformations of the plane. He does this by constructing a hyperbolic structure in Vessiot normal coordinates associated to this group and then transforming the resulting hyperbolic structure to the Vessiot distribution of equation (5.1). We can demonstrate this association of the Lie group $G_{3}$ with equation (5.1) directly by computing the tangential characteristic symmetries. The second order invariants of equation (5.1) may be easily computed from its characteristic systems; they are $u_{0}=r / \sqrt{4 p}+\sqrt{p} /(x+y)$ and $-v_{0}=$ $t / \sqrt{4 q}+\sqrt{q} /(x+y)$ for $F_{2}^{(2)}$ and $F_{1}^{(2)}$, respectively. A tedious but straightforward computation shows that the tangential characteristic symmetries of $F_{2}^{(2)}$ have the form

$$
V=a_{1}\left(x, u_{0}\right) \partial_{z}+\alpha_{2}\left(x, u_{0}\right) \partial_{\bar{p}}+a_{3}\left(x, u_{0}\right)\left(\frac{\partial_{\bar{p}}+\partial_{\bar{q}}}{x+y}-2 \bar{p} \partial_{z}\right),
$$

where $a_{1}, a_{2}, a_{3}$ are arbitrary functions and $\bar{p}=\sqrt{p}, \bar{q}=\sqrt{q}$, verifying Theorem 4.1a for this example. The Lie algebra of tangential characteristic symmetries is therefore spanned by

$$
V_{1}=\partial_{z}, \quad V_{2}=\partial_{\bar{p}}, \quad V_{3}=\frac{\partial_{\bar{p}}+\partial_{\bar{q}}}{x+y}-2 \bar{p} \partial_{z} .
$$

Fixing a leaf of the foliation by the joint level sets of the invariants (where, in particular, $x$ and $y$ are constants), we make a (leaf dependent) change of basis in the Lie algebra spanned by $V_{1}, V_{2}, V_{3}: V_{1} \mapsto V_{1}, V_{2} \mapsto V_{2}, V_{3} \mapsto V_{3}-(x+y)^{-1} V_{2}$, and then a change of variable: $w_{1}=z, w_{2}=-\bar{p}, w_{3}=2(x+y) \bar{q}$ transforming the tangential characteristic symmetries to the Lie algebra spanned by

$$
L_{1}=\partial_{w_{1}}, \quad L_{2}=\partial_{w_{2}}, \quad L_{3}=\partial_{w_{3}}+w_{2} \partial_{w_{1}} .
$$

This is precisely the Lie algebra which appears in Vessiot's hyperbolic structure for equation (5.1), being a basis for the right invariant vector fields (infinitesimal generators of left translations) on $G_{3}$, which is what we wanted to show. A similar computation can be carried out for the tangential characteristic symmetries of $F_{1}^{(2)}$. Finally, we see that it is possible to construct Vessiot normal coordinates for the hyperbolic structure by seeking normal forms for the tangential characteristic symmetries.

Example 5.3. So far we have only given examples of equations that are Darboux integrable on the 2-jets and all of these have been classified by Goursat and Vessiot. 
Theorems 4.1a and 4.1b allow us, in principle, to construct equations Darboux integrable on the $k$-jets for any $k$. The problem of how this may be done at any given order $k$ will require us to know, in particular, which Lie groups can appear as tangential characteristic symmetries. Given such a Lie group, this defines a family of manifolds of hyperbolic type parametrized by functions $\phi_{\alpha}$ and $\psi_{\alpha}$ as given by Theorems $4.1 \mathrm{a}$ and $4.1 \mathrm{~b}$. It may be that not all of these manifolds are the differential equations manifolds of an equation in $E$. The problem of deciding on these questions any further at this point will take us too far a field. For now we will be content to give an example of an equation which is Darboux integrable on a higher order jet and verify Theorem 4.1a in this case. In Vess42, Vessiot showed that the linear equations in $E$ which are $(2,2)$-Darboux integrable on the 2 -jets are associated with the three dimensional abelian Lie group, $\mathbb{R}^{3}$. That is, he showed that if $\mathcal{H}$ is the hyperbolic structure for a linear equation $(2,2)$-Darboux integrable equation on the 2-jets, then $\mathcal{H}$ may be expressed in terms of the left and right invariant vector fields on $\mathbb{R}^{3}$, that is, $\partial_{w_{1}}, \partial_{w_{2}}, \partial_{w_{3}}$. It is not difficult to verify that the equation

$$
u_{x y}+\frac{6}{(x+y)^{2}} u=0,
$$

is $(2,2)$-Darboux integrable on the 3 -jets. We prove that its tangential characteristic symmetries consist of the Lie group $\mathbb{R}^{5}$ and verify the structure of the corresponding manifolds of hyperbolic type in accordance with Theorem 4.1a We will compute the tangential characteristic symmetries of $F_{1}^{(3)}$ for equation (5.2). The differential equations manifold is 9-dimensional and a calculation shows the derived flag of $F_{2}^{(3)}$ stabilizes in dimension 7 . It therefore has 2 invariants and a calculation shows that these are $x$ and $\xi=u_{111}+g(x+y)^{-1} u_{11}+6(x+y)^{-2} u_{1}$ where we have used an obvious notation to denote the jet bundle coordinates in this case. From the symmetry of the equation it is obvious that the characteristic invariants of $F_{1}^{(3)}$ are $y$ and $\eta=u_{222}+6(x+y)^{-1} u_{22}+6(x+y)^{-2} u_{2}$. This verifies the Darboux integrability of equation (5.2). Including the characteristic invariants among new coordinates on the differential equations manifold $\mathcal{R}_{3}$ and making the change of variable: $p_{1}=u, p_{2}=u_{1}, p_{3}=u_{2}+(x+y) u_{22} / 2, p_{4}=u_{11}, p_{5}=-(x+y) u_{22} / 2$, transforms $F_{1}^{(3)}$ to the more convenient form

$$
\begin{aligned}
\{X= & \partial_{s}+p_{2} \partial_{p_{1}}+p_{4} \partial_{p_{2}}+s^{-1}\left(3 p_{3}+2 p_{5}\right) \partial_{p_{3}} \\
& \left.+\left(\xi-6 s^{-1} p_{4}-6 s^{-2} p_{2}\right) \partial_{p_{4}}-s^{-1}\left(3 p_{3}+2 p_{5}-6 s^{-1} p_{1}\right) \partial_{p_{5}}, \partial_{\xi}\right\}
\end{aligned}
$$

where $s=(x+y)$. In these coordinates, the tangential characteristic symmetries have the form $V=\sum_{i=1}^{5} V^{i} \partial_{p_{i}}$, where the $V^{i}$ are functions on $\mathcal{R}_{3}$. The equations $[V, X]=\left[V, \partial_{\xi}\right]=0$ become the equations $X V^{1}=V^{2}, X V^{2}=V^{4}, X V^{3}=$ $s^{-1}\left(3 V^{3}+2 V^{5}\right), X V^{4}=-6 s^{-2} V^{2}-6 s^{-1} V^{4}, X V^{5}=-s^{-1}\left(6 V^{1}-3 V^{3}-2 V^{5}\right)$ and $\partial_{\xi} V^{i}=0$ for all $i$. It is easily shown that the $V^{i}$ are independent of $p_{1}, \ldots, p_{5}$ and depend only upon $s, y$ and $\eta$ and these equations then become the (parametrized family of) ordinary differential equations,

$$
V_{s}^{i}=X V^{i},
$$

where the subscript $s$ denotes differentiation with respect to $s$ and where $y$ and $\eta$ are parameters. These equations form a standard system of ordinary differential equations to which the usual existence and uniqueness theorems apply. The space of solutions is therefore 5-dimensional and we conclude that on each joint level set 
of the characteristic invariants (where, in particular, $y$ and $\eta$ are constant), the infinitesimal tangential characteristic symmetries form a 5-dimensional Lie algebra. It is not hard to see that the Lie algebra is abelian and simply transitive. Indeed, let

$$
\phi^{1}(s)=\left(\phi_{j}^{1}(s)\right)_{j=1}^{5}, \ldots, \phi^{5}(s)=\left(\phi_{j}^{5}(s)\right)_{j=1}^{5},
$$

be a fundamental set for equations (5.3). Then if $V^{j}, j=1, \ldots, 5$, is any solution of (5.3) there are functions $c^{i}(t, \eta), i=1, \ldots, 5$, such that $V^{j}=\sum_{i} c^{i}(t, \eta) \phi_{j}^{i}(s)$. Hence, the tangential characteristic symmetries of equation (5.2) have the form

$$
V=\sum_{i, j=1}^{5} c^{i}(t, \eta) \phi_{j}^{i}(s) \partial_{p_{j}},
$$

and are therefore spanned by vector fields $V_{i}=\sum_{j} \phi_{j}^{i}(s) \partial_{p_{j}}, i=1, \ldots, 5$. The Lie algebra is clearly abelian and since matrix $\left(\phi_{j}^{i}(s)\right)$ is nonsingular it follows that it is also simply transitive. Indeed on each joint level set it is spanned by the vector fields $\partial_{p_{1}}, \partial_{p_{2}}, \ldots, \partial_{p_{5}}$ which is what we wanted to show. Moreover, it is easy to see that the (leaf dependent) change of variable $\bar{p}_{l}=\sum_{k} \psi_{l}^{k} p_{k}, l=1, \ldots, 5$, where $\left(\psi_{l}^{k}(s)\right)$ is the inverse of the fundamental matrix of equation (5.3), $\left(\phi_{k}^{i}(s)\right)$, rectifies the symmetries $V_{i}: \partial_{\bar{p}_{1}}, \ldots, \partial_{\bar{p}_{5}}$ reducing them to Vessiot normal form as we did in Example 5.2. By the invariance of the equation under the interchange of $x$ and $y$ we easily deduce that every tangential characteristic symmetry of $F_{2}^{(3)}$ for equation (5.2) has the form

$$
W=\sum_{i=1}^{5} d^{i}(x, \xi) V_{i},
$$

for arbitrary functions $d^{i}(x, \xi)$. This verifies Theorem 4.1a for this example.

\section{LOCAL EQUiVALENCE PROBLEM FOR (2,2)-DARBOUX INTEGRABLE EQUATIONS}

In this section, as a first application of tangential characteristic symmetries, we remark on the local equivalence problem for manifolds of $(2,2)$-hyperbolic type. This is very closely linked to the problem of deciding when two equations in $E$ that are $(2,2)$-Darboux integrable be locally equivalent under a contact transformation. The tangential characteristic symmetries are key invariants for this equivalence problem. This problem is, of course, a vast one. We will content ourselves with computing the form of the maximal class of local equivalences for manifolds of $(2,2)$-hyperbolic type.

Definition 6.1. Let $\mathcal{M}=\left(M_{n+4}, \mathcal{H}\right)$ and $\bar{M}=\left(\bar{M}_{n+4}, \overline{\mathcal{H}}\right)$ be a pair of manifolds of $(2,2)$-hyperbolic type and tangential characteristic symmetries $G$ and $\bar{G}$, respectively. We say that $\mathcal{M}$ and $\overline{\mathcal{M}}$ are locally equivalent if there is a local diffeomorphism $\tau: M \rightarrow \bar{M}$ which identifies their corresponding hyperbolic structures: $\tau_{*} H=\bar{H}$.

If $G$ is an $n$-dimensional Lie group, denote the Lie algebra of right invariant vector fields (infinitesimal left-translations) by $L_{1}, L_{2}, \ldots, L_{n}$ and the Lie algebra of left invariant vector fields (infinitesimal right translations) by $R_{1}, R_{2}, \ldots, R_{n}$. Denote left translation by $g \in G$ by $\lambda_{g}$ and right translation by $\rho_{g}$. 
Theorem 6.2. A pair of manifolds $\mathcal{M}$ and $\overline{\mathcal{M}}$ of (2,2)-hyperbolic type are locally equivalent if and only if:

(i) Their tangential characteristic symmetries, $G$ and $\bar{G}$, are isomorphic.

(ii) Given that $\mathcal{M}$ and $\overline{\mathcal{M}}$ are expressed in Vessiot normal coordinates, there is a transformation $\tau_{0} \in \mathcal{I} \times \mathcal{J}$ or $\tau_{0} \circ \sigma$ which identifies their respective hyperbolic structures, where $\mathcal{I} \times \mathcal{J}$ denotes the pseudogroup of automorphisms of the bundle $\mathbb{R}^{4} \times G \rightarrow \mathbb{R}^{4}$ of the form

$\left(u, u_{0}, v, v_{0}, w_{1}, \ldots, w_{n}\right) \rightarrow\left(A^{1}\left(u, u_{0}\right), A^{3}\left(u, u_{0}\right), A^{2}\left(v, v_{0}\right), A^{4}\left(v, v_{0}\right), W_{\alpha}\right)$,

where,

$$
W_{\alpha}=\lambda_{a\left(u, u_{0}\right)} \circ \rho_{b\left(v, v_{0}\right)} w_{\alpha},
$$

and $\sigma: \mathbb{R}^{4} \rightarrow \mathbb{R}^{4}$ is defined by $\sigma\left(u, u_{0}, v, v_{0}\right)=\left(\bar{u}\left(v, v_{0}\right), \bar{u}_{0}\left(v, v_{0}\right), \bar{v}\left(u, u_{0}\right)\right.$, $\left.\bar{v}_{0}\left(u, u_{0}\right)\right)$. Except that they define local diffeomorphisms, the smooth functions $A^{i}, a, b, \bar{u}, \ldots, \bar{v}_{0}$ are arbitrary.

Proof. We begin with an important observation, namely, the summands of a hyperbolic structure are invariant under local diffeomorphisms. More precisely, we have

Lemma 6.3. Let $\tau$ be an equivalence of manifolds $\mathcal{M}$ and $\overline{\mathcal{M}}$ of hyperbolic type, with hyperbolic structures $\mathcal{H}=\mathcal{H}_{1} \oplus \mathcal{H}_{2}$ and $\overline{\mathcal{H}}=\overline{\mathcal{H}}_{1} \oplus \overline{\mathcal{H}}_{2}$ respectively. Then either $\overline{\mathcal{H}}_{1}=\tau_{*} \mathcal{H}_{1}, \overline{\mathcal{H}}_{2}=\tau_{*} \mathcal{H}_{2}$, or $, \overline{\mathcal{H}}_{1}=\tau_{*} \mathcal{H}_{2}, \overline{\mathcal{H}}_{2}=\tau_{*} \mathcal{H}_{1}$.

Proof. This follows from the structure equations and the fact that the degree of a vector field is invariant under local diffeomorphisms 10

Continue with $\tau$ as an equivalence of manifolds $\mathcal{M}$ and $\overline{\mathcal{M}}$. Then by Lemma 6.3, $\tau$ induces an identification of the summands $\mathcal{H}_{i}$ and $\overline{\mathcal{H}}_{j}$ in one of two ways: $\tau_{*} \mathcal{H}_{i}=\overline{\mathcal{H}}_{j}$ with either $(i, j)=(1,1)$ and $(2,2)$, or, $(i, j)=(1,2)$ and $(2,1)$. Taking the first of these two possibilities implies that there are $G L(2)$-valued functions $g$ and $h$ on $M_{n+4}$ such that

$$
\tau_{*}\left(\begin{array}{c}
X_{1} \\
\partial_{u_{0}}
\end{array}\right)=g\left(\begin{array}{c}
\bar{X}_{1} \\
\partial_{\bar{u}_{0}}
\end{array}\right), \quad \tau_{*}\left(\begin{array}{c}
X_{2} \\
\partial_{v_{0}}
\end{array}\right)=h\left(\begin{array}{c}
\bar{X}_{2} \\
\partial_{\bar{v}_{0}}
\end{array}\right),
$$

where $\mathcal{H}$ and $\overline{\mathcal{H}}$ are expressed in Vessiot normal coordinates. For convenience, we temporarily denote $u, u_{0}, v, v_{0}$ by $x_{1}, x_{3}, x_{2}, x_{4}$ respectively. The equivalence $\tau$ has the form $\tau(x, w)=\left(A^{1}(x, w), A^{3}(x, w), A^{2}(x, w), A^{4}(x, w), W_{\alpha}(x, w)\right), \alpha=$ $1,2, \ldots, n$. Then from the first equation in (6.1), we have

$$
\begin{aligned}
& \sum_{i=1}^{4}\left(X_{1} A^{i}\right) \partial_{\bar{x}_{i}}+\sum_{i=1}^{n}\left(X_{1} W_{\alpha}\right) \partial_{W_{\alpha}}=g_{11} \bar{X}_{1}+g_{12} \partial_{\bar{x}_{3}}, \\
& \sum_{i=1}^{4}\left(\partial_{x_{3}} A^{i}\right) \partial_{\bar{x}_{i}}+\sum_{i=1}^{n}\left(\partial_{x_{3}} W_{\alpha}\right) \partial_{W_{\alpha}}=g_{21} \bar{X}_{1}+g_{22} \partial_{\bar{x}_{3}} .
\end{aligned}
$$

These equations and the other two arising from the second equation in (6.1) show that $A^{1}, A^{3}$ are invariants of $\mathcal{H}_{2}$ and $A^{2}, A^{4}$ are invariants of $\mathcal{H}_{1}$ and hence we have $A^{1}=A^{1}\left(u, u_{0}\right), A^{3}=A^{3}\left(u, u_{0}\right), A^{2}=A^{2}\left(v, v_{0}\right), A^{4}=A^{4}\left(v, v_{0}\right)$. In accordance

\footnotetext{
${ }^{10}$ Remark that Lemma 6.3 together with Proposition 2.6 give a formal proof of the contact invariance of hyperbolicity for second order scalar partial differential equation in two independent variables.
} 
with Theorem 4.1a, let $\mathcal{L}=\left\{L_{\alpha}\right\}, \overline{\mathcal{L}}=\left\{\bar{L}_{\alpha}\right\}$ denote bases for the tangential characteristic symmetries of $\mathcal{H}_{2}$ and $\overline{\mathcal{H}}_{2}$, respectively, and $\mathcal{R}=\left\{R_{\alpha}\right\}, \overline{\mathcal{R}}=\left\{\bar{R}_{\alpha}\right\}$ those of $\mathcal{H}_{1}$ and $\overline{\mathcal{H}}_{1}$ respectively. If $V$ is a tangential characteristic symmetry of $\mathcal{H}_{1}$, then $\tau_{*} V$ is a tangential characteristic symmetry of $\tau_{*} \mathcal{H}_{1}=\overline{\mathcal{H}}_{1}$. However, by Theorem 4.1a, we know that every tangential characteristic symmetry of $\overline{\mathcal{H}}_{1}$ has the form $s_{\alpha}\left(\bar{v}, \bar{v}_{0}\right) \bar{R}_{\alpha}$. Hence there is a $G L(n)$-valued function $\left(s_{\alpha \beta}\left(\bar{v}, \bar{v}_{0}\right)\right)$ such that $\tau_{*} R_{\alpha}=s_{\alpha \beta}\left(\bar{v}, \bar{v}_{0}\right) \bar{R}_{\beta}$. Similarly, there is a $G L(n)$-valued function $\left(t_{\alpha \beta}\left(\bar{u}, \bar{u}_{0}\right)\right)$ such that $\tau_{*} L_{\alpha}=t_{\alpha \beta}\left(\bar{u}, \bar{u}_{0}\right) \bar{L}_{\alpha}$. Thus, $\tau$ identifies the tangential characteristic symmetries of $\mathcal{M}$ and $\overline{\mathcal{M}}: \tau_{*} \mathcal{L}=\overline{\mathcal{L}}, \tau_{*} \mathcal{R}=\overline{\mathcal{R}}$. By standard results this implies that $G$ and $\bar{G}$ are isomorphic, proving the necessity of part (i). We now easily deduce that an equivalence $\tau$ implies the existence of functions $a, b, c, d$ such that

$$
\begin{array}{ll}
\frac{\partial W_{\alpha}}{\partial u} \partial_{W_{\alpha}}=a^{\alpha}\left(u, u_{0}\right) L_{\alpha_{\left.\right|_{\tau(x, w)}}}, & \frac{\partial W_{\alpha}}{\partial u_{0}} \partial_{W_{\alpha}}=b^{\alpha}\left(u, u_{0}\right) L_{\left.\alpha\right|_{\tau(x, w)}}, \\
\frac{\partial W_{\alpha}}{\partial v} \partial_{W_{\alpha}}=c^{\alpha}\left(v, v_{0}\right) R_{\alpha_{\left.\right|_{\tau(x, w)}}}, & \frac{\partial W_{\alpha}}{\partial v_{0}} \partial_{W_{a}}=d^{\alpha}\left(v, v_{0}\right) R_{\alpha_{\left.\right|_{\tau(x, w)}}} .
\end{array}
$$

We proceed to characterize the most general solution $\left(W_{1}(x, w), \ldots, W_{n}(x, w)\right)$ of (6.3) and (6.4). This follows from

Lemma 6.4. Let $G$ be an $n$-dimensional Lie group and $\left\{L_{1}, \ldots, L_{n}\right\}$ a basis for the Lie algebra of infinitesimal left translations on $G$. Fix functions $A_{\alpha}\left(t_{1}, t_{2}\right)$, $B_{\alpha}\left(t_{1}, t_{2}\right), \alpha=1, \ldots, n$, which are smooth in a domain $U \subset \mathbb{R}^{2}$ containing $(0,0)$. Consider the initial value problem

$$
\Delta_{*}\left(\partial_{t_{1}}\right)=A_{\alpha}\left(t_{1}, t_{2}\right) L_{\alpha}, \quad \Delta_{*}\left(\partial_{t_{2}}\right)=B_{\alpha}\left(t_{1}, t_{2}\right) L_{\alpha}, \quad \Delta(0)=x_{0},
$$

for a map $\Delta: U \rightarrow G$. Then provided the integrability conditions are satisfied in (6.5), this problem has a unique solution

$$
\Delta\left(t_{1}, t_{2}\right)=\lambda_{a\left(t_{1}, t_{2}\right)} x_{0}
$$

where $\left(t_{1}, t_{2}\right) \mapsto \lambda_{a\left(t_{1}, t_{2}\right)}$, is a unique family of left-translations on $G$ satisfying $\lambda_{a(0,0)}=i d_{G}$.

Proof. Substitute the proposed solution (6.6) into the initial value problem (6.5) and use the right invariance of the $L_{\alpha}$. Then use the existence and uniqueness of solutions to completely integrable Mayer-type systems.

Remark. This lemma is a slight generalization of the classically known systems of Lie type; (see Bryant [B] , Lecture 3, Proposition 3). The main point is that every solution of the differential equations in (6.5) has the form (6.6). So for Mayer systems of Lie type we have a precise notion of general solution analogous to the notion of general solution for linear ordinary differential equations which are a special case of these general systems.

We see from Lemma 6.4 that, provided integrability conditions are satisfied, "along" $u=u_{0}=0$, the general solution of system (6.4) is a map $\Delta_{1}: \mathbb{R}^{2} \times G \rightarrow G$ given by $\Delta_{1}\left(v, v_{0} ; z_{0}\right)=\rho_{b\left(v, v_{0}\right)} z_{0}$. Similarly, along $v=v_{0}=0$, the general solution of system (6.3) is a map $\Delta_{2}: \mathbb{R}^{2} \times G \rightarrow G$ given by $\Delta_{2}\left(u, u_{0} ; z_{1}\right)=\lambda_{\alpha\left(u, u_{0}\right)} z_{1}$, for unique families of, respectively, right- and left-translations $\rho_{b\left(v, v_{0}\right)}, \lambda_{a\left(u, u_{0}\right)}$ satisfying $\rho_{b(0,0)}=\lambda_{a(0,0)}=\operatorname{id}_{G}$. It is easy to show that for all $\left(v, v_{0}\right),\left(u, u_{0}\right)$ sufficiently close to $(0,0), \rho_{b\left(v, v_{0}\right)} \circ \Delta_{2}$ is a solution of system (6.3) and $\lambda_{a\left(u, u_{0}\right)}{ }^{\circ} \Delta_{1}$ is a solution of system (6.4) owing to the right-invariance of the $L_{\alpha}$ and left-invariance of the 
$R_{\alpha}$. But these two functions are equal since left- and right-translations commute and hence the map $\Delta: \mathbb{R}^{4} \times G \rightarrow G$ defined by

$$
\Delta\left(u, u_{0}, v, v_{0} ; w\right)=\lambda_{a\left(u, u_{0}\right)} \circ \rho_{b\left(v, v_{0}\right)} w,
$$

satisfies both systems (6.3) and (6.4). On the other hand, systems (6.3) and (6.4) constitute a completely integrable Mayer system and hence by the existence and uniqueness theorem for such systems (the Frobenius theorem), there is exactly one solution passing through the point $(0,0,0,0, w) \in \mathbb{R}^{4} \times G$. Consequently, the family of solutions (6.7) (parametrized by the points of $G$ ) accounts for every solution of systems (6.3) and 6.4).

Consider now the other possibility for the action of an equivalence $\tau$ in accordance with Lemma (6.3), namely, $\tau_{*} \mathcal{H}_{1}=\overline{\mathcal{H}}_{2}, \tau_{*} \mathcal{H}_{2}=\overline{\mathcal{H}}_{1}$. Then an entirely analogous argument to the one given confirms the necessary form of the equivalence in this final case.

Conversely, if $G$ and $\bar{G}$ are isomorphic, then by Theorem 4.1a we may express both the hyperbolic structures $\mathcal{H}$ and $\overline{\mathcal{H}}$ in terms of left- and right-invariant vector fields on $G$. The pseudogroup $\mathcal{I} \times \mathcal{J}$ is then defined and the identification guaranteed by part (ii) defines an equivalence of manifolds of $(2,2)$-hyperbolic type.

Corollary 6.5. A pair of (2,2)-Darboux integrable equations are locally contact equivalent if and only if their corresponding manifolds of hyperbolic type are locally equivalent. In particular, the isomorphism class of the tangential characteristic symmetries is a contact invariant.

Proof. The hyperbolic structure of a differential equation is diffeomorphic to its Vessiot distribution.

Corollary 6.5 makes clear the fact that the Liouville equation and the Goursat equation (5.1) are not contact equivalent since they have nonisomorphic tangential characteristic symmetries. The Liouville equation and the two equations

$$
u_{x y}=\frac{\sqrt{u_{x}^{2}-1} \sqrt{u_{y}^{2}-1}}{\sinh u}, \quad u_{x y}=e^{u} \sqrt{u_{x}^{2}-1},
$$

each have tangential characteristic symmetries isomorphic to $S L(2, \mathbb{C})$. However, as Vessiot shows Vess42, their hyperbolic structures are distinct. These three equations are therefore contact inequivalent. Indeed, they appear on Goursat's list as representatives of three contact orbits of equations in $E,(2,2)$-Darboux integrable on the 2-jets. We observe, finally, that the tangential characteristic symmetries distinguish between the Liouville equation and the linear equation

$$
u_{x y}=\frac{2 u}{(x+y)^{2}},
$$

which, like the Liouville equation, is (2,2)-Darboux integrable on the 2-jets. The tangential characteristic symmetries of the later are isomorphic to the abelian Lie group $\mathbb{R}^{3}$. Of course, all the results of this section may be formulated for $(2,3)$ Darboux integrable equations in a similar manner. However, as this section is meant to serve only as a pointer to the possible results concerning tangential characteristic symmetries, we will not discuss the details of this here.

Remark. Corollary 6.5] addresses the intrinsic equivalence problem for this class of equations which is the notion of equivalence that we are using in this paper. This 
is natural from the point of view of differential equations since there is a bijection between the solutions of two intrinsically equivalent differential equations. There is another notion, that of extrinsic equivalence. Two differential equations (say) $\mathcal{R}$ and $\overline{\mathcal{R}} \subset J^{2}$ are extrinsically equivalent if there is a contact transformation $\rho: J^{2} \rightarrow J^{2}$ whose restriction to $\mathcal{R}$ has image $\overline{\mathcal{R}}$. In general the two notions of intrinsic and extrinsic equivalence are distinct as was pointed out by Cartan in [Ca]. However, a result of Gardner and Kamran [GK], shows that for second order scalar partial differential equations in two independent variables, there is a one-to-one correspondence between the solutions of the intrinsic and extrinsic equivalence problems. Hence, for the equations treated in this paper, there is no loss of generality in considering only intrinsic equivalence (see also [AKO]).

\section{ACKNOWLEDGEMENTS}

A substantial portion of the research for this paper was carried out while I was a Visiting Fellow at the Centre for Mathematics and its Applications at the Australian National University. It is a pleasure to thank the CMA for its kind hospitality. I would like to thank Philip Doyle for his insightful suggestion that I study symmetries of characteristic systems and for extensive conversations on the geometry of differential equations. Finally, I would like to thank Niky Kamran, David Hartley and Philip Doyle for reading an earlier draft of this paper and for making valuable suggestions.

\section{REFERENCES}

[AK] I. M. Anderson and N. Kamran, The variational bicomplex for hyperbolic second-order scalar partial differential equations in the plane, Duke Math. J. 87 (2) (1997), 265-319. MR 98e:58183

[AKO] I. M. Anderson, N. Kamran, and P. J. Olver, Internal, external and generalized symmetries, Adv. Math. 100 (1993), 53-100. |MR 94g:58257|

[B] R. L. Bryant, An Introduction to Lie Groups and Symplectic Geometry, IAS/Park City Mathematics Series, Amer. Math. Soc., Vol. 1, 1995. MR 96i:58002

[BH] R. L. Bryant and L. Hsu, Rigidity of integral curves of rank 2 distributions, Invent. Math. 114 (1993), 435-461. MR 94j:58003

[BGH] R. L. Bryant and P. A. Griffiths, and L. Hsu, Hyperbolic exterior differential systems and their conservation laws I, II, Selecta Math. (N.S.) 1 (1995), 21-112, 265-323. MR 97d:58008 MR 97d:58009

[BC3G] R. L. Bryant, S. S. Chern, R. B. Gardner, H. L. Goldschmidt, P. A. Griffiths, Exterior Differential Systems, Springer-Verlag, 1991. MR 92h:58007

[Ca] É. Cartan, Les systèmes de Pfaff á cinq variables et les équations aux dérivées partielles du seconde ordre, Ann. Sci. École Norm. (3) 27 (1910), 109-192.

[CH] R. Courant and D. Hilbert, Methods of Mathematical Physics, Vols. 1,2, Wiley, 1962. MR 25:4216

[Ga] R. B. Gardner, A differential geometric generalization of characteristics, Comm. Pure Appl. Math. 22 (1969), 597-626. MR 41:618

[GK] R. B. Gardner and N. Kamran, Characteristics and the geometry of hyperbolic equations in the plane, J. Differential Equations 104 (1993), 60-116. MR 94h:58006]

[Go96] E. Goursat, Leçons sur l'intégration des équations aux dérivées partielles du second order á deux variables indépendent, Tome 1, Hermann, Paris, 1896.

[Go99] E. Goursat, Recherches sur quelques équation aux dérivées partielles du second ordre, Ann. Fac. Toulouse $2^{e}$ serie (1899), 31-68, 439-464.

[HsK] L. Hsu and N. Kamran, Classification of second-order ordinary differential equations admitting Lie groups of fibre preserving point symmetries, Proc. Lond. Math. Soc. 58 (1989), 387-416. MR 89k:58312 
[JA] M. Juráš and I. M. Anderson, Generalized Laplace invariants and the method of Darboux, Duke Math. J. 89 (2) (1997), 351-375. MR 98h:58004

[K] N. Kamran, Contributions to the study of the equivalence problem of Élie Cartan and its applications to partial and ordinary differential equations, Mem. Cl. Sci. Acad. Roy. Belg. 45 (1989), Fac. 7. MR 92h:58008

[KV] I. S. Krasil'shchik and A. M. Vinogradov (Eds.), Symmetries and Conservation Laws for Differential Equations of Mathematical Physics, Translations of Mathematical Monographs, Vol. 182, Amer. Math. Soc. 1999. CMP 99:08

[Lie] S. Lie, Diskussion der differential gleichung $s=F(z)$, Archiv. für Math. Bd. VI Heft 2 (1881), 112-124.

[Ne] A. C. Newell, Solitons in Mathematics and Physics, SIAM, CBMS-NSF, Vol. 48, 1985. MR 87h:35314

[Pa] R. S. Palais, The symmetries of solitons, Bull. Amer. Math. Soc. 34 (4) (1997), 339-403. MR 98f:58111

[Sh] R. W. Sharpe, Differential Geometry: Cartan's Generalization of Klein's Erlangen Program Graduate Texts in Mathematics, Vol. 166, Springer 1997. MR 98m:53033

[Va90] P. J. Vassiliou, Equivalence of vector field systems, Bull. Austral. Math. Soc. 42 (2) (1990), 215-229.

[Va94] P. J. Vassiliou, On some geometry associated with a generalized Toda lattice, Bull. Austral. Math. Soc. 49 (3) 439-462. MR 95i:58097

[Va99] P. J. Vassiliou, Intrinsic geometry of systems of first order partial differential equations, School of Mathematics and Statistics, University of Canberra, 1999, preprint.

[Vess24] E. Vessiot, Sur une théorie nouvelles des problèmes généraux d'intégration, Bull. Soc. Math. Fr. 52 (1924), 336-395.

[Vess36] E. Vessiot, Sur les faisceaux de transformations infinitésimales associées aux équations aux dérivées partielles du second ordre, $F(x, y, z, p, q, r, s, t)=0$, J. Math. Pures Appl. 25 (1936), 301-320.

[Vess39] E. Vessiot, Sur les équations aux dérivées partielles du second ordre, $F(x, y, z, p, q, r, s, t)$ $=0$, intégrables par la methode de Darboux, J. Math. Pure Appl. 18 (9), 1-61.

[Vess42] E. Vessiot, Sur les équations aux dérivées partielles du second ordre, $F(x, y, z, p, q, r, s, t)$ = , intégrables par la methode de Darboux (suite), J. Math. Pure Appl. 21 (9) (1942), 1-66. MR 5:679

School of Mathematics and Statistics, University of Canberra, Canberra ACT, AusTRALIA, 2601

E-mail address: pierre@ise.canberra.edu.au 